\title{
Attention misallocation, social welfare and policy implications
}

\author{
Luo, Yulei ; Chen, Heng ; Pei, Guangyu
}

\begin{abstract}
We examine how agents allocate attention between private and public signals to reduce the uncertainty about observation noises when coordination is an important concern. In this setting, the attention allocation may not be monotone in endowed attention capacity. Agents may decrease their attention on or even ignore the more accurate signal when capacity increases. As a result, social welfare may decrease when they have more attention to process information. And it can be even higher when agents possess a finite amount of capacity than when they have an infinite amount of capacity. We derive sufficient and necessary conditions under which multiple equilibria emerge and study the implications of equilibrium multiplicity for macroeconomic policies.
\end{abstract}

DOI: https://doi.org/10.1016/j.jedc.2015.05.003

Posted at the Zurich Open Repository and Archive, University of Zurich ZORA URL: https://doi.org/10.5167/uzh-110798

Journal Article Accepted Version

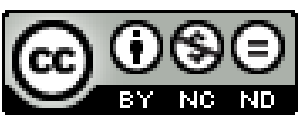

The following work is licensed under a Creative Commons: Attribution-NonCommercial-NoDerivatives 4.0 International (CC BY-NC-ND 4.0) License.

Originally published at:

Luo, Yulei; Chen, Heng; Pei, Guangyu (2015). Attention misallocation, social welfare and policy implications. Journal of Economic Dynamics and Control, 59:37-57.

DOI: https://doi.org/10.1016/j.jedc.2015.05.003 


\title{
Attention Misallocation, Social Welfare and Policy Implications *
}

\author{
HENG CHEN ${ }^{\dagger}$ \\ University of Hong Kong \\ YULEI LUO $¥$ \\ University of Hong Kong \\ GUANGYU PEI ${ }^{\S}$ \\ University of Zurich
}

April 21, 2015

\begin{abstract}
We examine how agents allocate attention between private and public signals to reduce the uncertainty about observation noises when coordination is an important concern. In this setting, the attention allocation may not be monotone in endowed attention capacity. Agents may decrease their attention on or even ignore the more accurate signal when capacity increases. As a result, social welfare may decrease when they have more attention to process information. And it can be even higher when agents possess a finite amount of capacity than when they have an infinite amount of capacity. We derive sufficient and necessary conditions under which multiple equilibria emerge and study the implications of equilibrium multiplicity for macroeconomic policies.
\end{abstract}

Keywords. Coordination game, social welfare, rational inattention

JEL Classification. C72, D60, E58

\footnotetext{
${ }^{* \dagger}$ Chen: Corresponding author, hengchen@hku.hk, Tel: 00852-28578506, K.K. Leung Building, The University of Hong Kong, Pokfulam Road, Hong Kong; ${ }^{\ddagger}$ Luo:yluo@econ.hku.hk, K.K. Leung Building, The University of Hong Kong, Pokfulam Road, Hong Kong; and \$Pei:guangyu.pei@uzh.ch; Schonberggasse 1, CH-8001 Zurich. We thank George-Marios Angeletos, Melody Lo, Jun Nie, Alessandro Pavan, Wing Suen, and Satoru Takahashi. We also thank seminar participants at Second Conference on Rational Inattention and Related Theories and 19th International Conference of Computing in Economics and Finance for their helpful comments. Luo thanks the Hong Kong General Research Fund (\#HKU791913) for financial support.
} 


\section{Introduction}

Coordination games with heterogeneity in information and complementarity in action have been widely applied to macroeconomic environments, financial markets and even collective actions. In this paper, we study a scenario, where agents that play such a coordination game have only limited capacity to process relevant information. Consequently, they have to allocate their capacity optimally among various information sources and then take actions based on the information they acquire. As Sims (2005) argue, this information processing constraint may have significant welfare implications for understanding the effects of policies that reveal public information and can be critical when evaluating the optimality of policies, e.g., the transparency of public announcements. ${ }^{1}$

To abstract from a specific market structure and retain tractability, we formalize our model in a "beauty contest" framework, as in Morris and Shin (2002), where the payoff for an individual depends on the distance of his action from an unobservable state and from the average action. To take the best action, agents must estimate the underlying state and forecast the average action of others. There are two correlated signals that reveal noisy information about the fundamental, and they can be observed if agents pay attention to them. One of the signals is private and contains idiosyncratic noise, and the other is public, can be potentially observed by all agents and contains common noise. The main point of departure of our model is to assume that agents cannot perfectly observe these signals because they possess a limited capacity to process information. Consequently, agents can only observe these signals with idiosyncratic observation noises.

This setting has captured the essence of several important macroeconomic environments and financial markets. For example, consider a price setting model with monopolistic competition in an informationally segregated island economy. Firms want to minimize the distance between their price and a target price that is the weighted average of an unknown state (e.g., log of nominal output) and the aggregate price. The representative firm on each island has access to public information about the nominal output (e.g., the central bank's guidance) and some island-specific information (e.g., local forecast). They can extract information about the state of the economy through those public and local sources, both of which reveal noisy information about the aggregate state, and are relevant for forecasting the aggregate price level. Constrained by a limited amount of capacity, firms need to decide on the allocation of their attention to process each of the noisy signals.

\footnotetext{
${ }^{1}$ Sims (2005) argues that "rational inattention may have far-reaching implications for macroeconomics and monetary policy generally, once its implications are fully worked out. In the meantime, though, it may shed some light on transparency in monetary policy."
} 
Another case in point is institutional investors in financial markets. They typically have access to information from a public source, e.g., announcements by the central bank or annual reports released by the firms whose securities are traded, and information from private sources, e.g., research reports on macro conditions or specific firms provided by analysts in research departments. Both types of sources reveal noisy information about the market fundamental. Investors need to allocate their limited attention optimally so that they can estimate the true fundamental and the aggregate action before making decisions.

Questions naturally arise. How do individuals allocate their attention between these two information sources? Under what conditions, do multiple equilibria emerge? Does social welfare necessarily increase when individuals possess more informationprocessing capacity?

In this model, a number of forces interact and shape agents' decisions: decreasing returns to attention, the relative accuracy of the public signal to the private signal, the coordination motive and the correlation between the two signals. ${ }^{2}$ It is more attractive for agents to observe the signal with higher accuracy, because a signal of higher quality helps agents estimate the state more accurately. However, the force of diminishing returns to attention provides agents with incentives to diversify their attention and spend their capacity on both signals. ${ }^{3}$ The coordination motive tilts the attention allocation decision toward the public signal, which better aligns their actions. Furthermore, a higher correlation across signals dampens the effect of diminishing returns to attention and amplifies the effect of the coordination motive.

The four factors combined may lead to the fact that agents allocate their attention in a non-monotone fashion, i.e., attention paid onto each signal may not necessarily increase in the total amount of capacity. For example, one intriguing scenario may arise, where agents can first focus on the relatively more precise private signal and then diversify their attention when capacity is higher; however, when there is a further increase in capacity, they may reduce their attention on or even ignore the private signal of higher quality and instead focus on the relatively imprecise public signal. We label this phenomenon "attention misallocation." Further, when the coordination

\footnotetext{
${ }^{2}$ Sims (2010) argues that finite capacity can be elastic in response to a change in environment, given that the marginal cost of information processing is constant. In this case, inattentive agents are allowed to adjust optimal capacity in such a way that the marginal cost of information-processing for the problem at hand remains constant, which is consistent with the concept of "elastic" capacity proposed in Kahneman (1973). Luo and Young (2014) notes that the two assumptions, i.e., constant capacity and constant marginal cost of information processing, are observationally equivalent in the sense that they lead to the same model dynamics governed by the Kalman gain. In this study, for simplicity, we focus on the fixed capacity assumption and do not consider the effect of prior uncertainty on elastic capacity.

3"Diminishing returns to attention" refers to the fact that the marginal increase in the agent's welfare is decreasing as capacity increases. Luo (2008) and Luo and Young (2010) illustrate this property in partial equilibrium permanent income models with inattentive agents.
} 
motive or correlation is sufficiently high, the relative accuracy is not extreme and the amount of capacity is not very high, multiple equilibria can arise in this model.

We also find a number of distinct results on social welfare, i.e., the average distance between individual decisions and the underlying state. First, social welfare may decrease when individuals possess more capacity to process information. This result hinges on the fact that agents may "misallocate" their attention from a social perspective and the misallocation may become more severe in response to higher capacity. When there is an increase in capacity, agents can observe signals more clearly and better estimate the underlying state. However, given the desire to align their actions, they may decrease the attention paid to the private signal, even though it is relatively more precise, and coordinate even more attention on the less precise public signal. When agents take action, they assign a larger weight to the observation of the public signal, which exacerbates the "overreaction" to the public signal and causes a decrease in social welfare. ${ }^{4}$

Second, the limit case of this model is the world of Morris and Shin (2002), in which agents possess an infinite amount of capacity and can therefore perfectly observe both signals. However, strikingly, social welfare in the Morris-Shin world may be even lower than that in our model with capacity-limited agents. On the one hand, with a finite amount of capacity, agents have a less precise estimation of the fundamental than that in the Morris-Shin world. On the other hand, agents may endogenously pay little attention to the public signal and therefore reduce their reliance on it in their action which, to a certain degree, alleviates the overuse of the public signal. We show that the second effect can dominate.

Third, our model also sheds some light on the debate about the transparency of monetary policy. Morris and Shin (2002) show that social welfare can decrease when the central bank delivers a clearer public announcement due to an overreaction to the public signal. Svensson (2006) questions the empirical relevancy of this result and argues that it only holds when public information is implausibly imprecise. We show that endogenous attention allocation can amplify the "overreaction," so that social welfare can decrease, even when the precision of the public signal is reasonably high.

Finally, our results also offer a new perspective on the literature covering the efficient use of information. Angeletos and Pavan (2007) show that equilibrium use and the efficient use of information coincide if and only if the social and private values of coordination are the same. However, once we allow for an endogenous information

\footnotetext{
${ }^{4}$ In the existing rational inattention literature, welfare typically increases in the capacity of processing information. For example, Luo (2008) shows that the welfare loss due to finite capacity decreases with channel capacity within a partial equilibrium permanent income model. Maćkowiak and Wiederholt (2011) obtains the same result in a general equilibrium business cycle model.
} 
structure, i.e., attention allocation of inattentive agents, this relationship breaks down.

From a theoretical point of view, we also contribute to the literature of rational inattention by characterizing attention allocation between two correlated signals. This is technically challenging because, in this case, some capacity has to be "wasted" to learn the correlated part twice, and it is difficult to separate the amount of capacity that effectively reduces the observation noise of one signal from the other. We have bypassed this difficulty by using a transformation in which we define "effective capacity" so that such a separation is feasible. The standard information processing constraint for independent signals becomes a special case in our formulation.

The rest of the paper is organized as follows. Section 2 discusses the related literature. Section 3 characterizes a beauty contest model with rational inattentive agents and examines the equilibrium properties of the model. Section 4 explores the attention allocation decision via comparative statics. Section 5 studies the social welfare implications of limited attention. Section 6 addresses policy issues that are discussed in the literature. Section 7 contains a discussion on the alternative information structures. The last section concludes.

\section{Related Literature}

There are two approaches to modeling information acquisition in the related literature: "costly acquisition" with the cost of acquiring information being convex in the precision of signals and "rational inattention" with a fixed amount of attention being split among signals. These two approaches captures two distinct aspects of learning. With the former, it is increasingly costly for agents to find additional evidence for the truth; with the latter, agents are in a process of increasingly directed or refined search for an answer when they spend more capacity in learning. ${ }^{5}$ In other words, the costly acquisition approach assumes that agents incur increasing marginal cost when they acquire more information, while the rational inattention approach assumes the marginal cost of information acquisition is decreasing.

Pioneering studies that adopt the costly acquisition approach examine the implications of information acquisition in coordination games, e.g., Hellwig and Veldkamp (2009), Myatt and Wallace (2012) and Yang (2013). Hellwig and Veldkamp (2009) show that there could exist multiple equilibria in their coordination model, because agents make a binary signal purchase decision and a public signal becomes more valuable when others also purchase it, due to strategic complementarity. Myatt and Wallace (2012) show that equilibrium is unique, even with complementarity in action, when

\footnotetext{
${ }^{5} \mathrm{~A}$ capacity can be considered approximately the number of binary signals that partition states of the world, and moreover, the interpretation of each signals depends on its predecessors, see Veldkamp (2011) for elaboration.
} 
agents can also choose the observation noises. In contrast, we follow Sims (2003) and assume that agents split a fixed amount of capacity on the signals to be observed. In our model, we demonstrate that multiplicity can arise, when the information acquisition is continuous and when learning takes the form of increasingly directed and refined search, captured by the rational inattention approach.

We intend to offer a welfare analysis of the coordination game played by rationally inattentive agents to study the effect that attention allocation has on social welfare. Our setup differs from those of recent contributions to the literature that explicitly deals with welfare-related issues. Maćkowiak and Wiederholt $(2009,2011)$ study how individuals or firms allocate their attention among two independent states when they set the price in a market-based economy or take collective actions. In our case, the two signals are correlated. We explicitly characterize the role of their correlation in optimal attention allocation and show that correlation is of critical importance for the multiplicity and uniqueness of equilibrium. For example, a high coordination motive does not necessarily give rise to multiple equilibria, unless the correlation between the two signals is sufficiently high.

This paper is closely related to the literature on the efficient use of information, e.g. Angeletos and Pavan (2006) and Colombo, Femminis, and Pavan (2012). The latter studies the interaction between the inefficient use and acquisition of information. In their model, agents pay to gain private information and can observe the public announcement precisely. In contrast, agents in our model can observe neither of the signals perfectly. Unlike their setup, which has a unique equilibrium, the rational inattention assumption in our model gives rise to the possibilities of multiple equilibria and of one of the signals being endogenously ignored.

Our work also contributes to the growing literature on the social value of public information. Cornand and Heinemann (2008) consider an interesting setup in which only a fraction of the agents are allowed to observe the public signal. In our model, agents can endogenously choose to ignore either public or private information, or diversify their attention between both. Myatt and Wallace (2009) study this issue in a model with multiple information sources that differ in the degree of publicity. In our model, the publicity of public information is endogenous: the idiosyncratic observation noise is determined by the amount of attention paid.

This paper is also broadly related to the literature on information choice, attention allocation and asset allocation, which includes Peng (2005), Peng and Xiong (2006), Nieuwerburgh and Veldkamp $(2009,2010)$ and Mondria $(2010)$. The framework adopted in these studies consists of multiple assets and a continuum of agents who face the information processing constraints. 


\section{The Model}

\subsection{Players, Payoffs and Coordination}

The economy is occupied by a continuum of agents, indexed by $i \in(0,1)$. Each of them can choose an action, $a_{i} \in R$. In this economy, the fundamental state, $\theta$, affects payoffs of agents. It is selected by nature but unknown to agents. Following Morris and Shin (2002), the payoff for agent $i$ is specified by

$$
u_{i}=-\left(a_{i}-\theta\right)^{2}-\frac{\alpha}{1-\alpha}\left(L_{i}-\bar{L}\right) \text {, }
$$

where $\alpha$ is constant, such that $0<\alpha<1$, and $L_{i}=\int\left(a_{j}-a_{i}\right)^{2} \mathrm{~d} j$ and $\bar{L}=\int L_{i} \mathrm{~d} i$.

When agent $i$ takes action, two types of loss are incurred. The first component is measured by the distance between individual action and the uncertain state: agents would be better off if they were to choose an action closer to the fundamental. The second component is the distance between individual and average actions, which captures the idea that agents want to align their actions. A higher $\alpha$ implies that agents assign a larger weight to this strategic concern in their payoff structure and have a stronger incentive to coordinate.

\subsection{Information Structure}

Agents begin with some knowledge of the underlying state. Specifically, they share a common normal prior over $\theta$,

$$
\theta \sim N\left(\bar{\theta}, \sigma^{2}\right)
$$

where $\bar{\theta}$ and $\sigma^{2}$ are the mean and variance of the prior distribution, respectively. Each agent can access two potentially observable signals, i.e., the private signal $x_{i}$ and the public signal $z$, and the distribution is specified as follows,

$$
s_{i}=\left(\begin{array}{c}
x_{i} \\
z
\end{array}\right) \triangleq\left(\begin{array}{c}
\theta+\varepsilon_{x_{i}} \\
\theta+\varepsilon_{z}
\end{array}\right),
$$

where $\varepsilon_{x_{i}} \sim N\left(0, \sigma_{x}^{2}\right)$ and $\varepsilon_{z} \sim N\left(0, \sigma_{z}^{2}\right)$ are independent of the true state $\theta$. Note that $\varepsilon_{x_{i}}$ is independently and identically distributed across agents while $\varepsilon_{z}$ is common. The ex ante covariance matrix of $s_{i}$ can be written as

$$
\Sigma=\left(\begin{array}{cc}
\sigma^{2}+\sigma_{x}^{2} & \sigma^{2} \\
\sigma^{2} & \sigma^{2}+\sigma_{z}^{2}
\end{array}\right) .
$$

The information structure described thus far resembles that in Morris and Shin 
(2002). The public signal can be interpreted as a public announcement made by the central bank or statistics released by the public agency. The private signal can be interpreted as information only accessible to individuals and not to the general public. Noise terms $\varepsilon_{x_{i}}$ and $\varepsilon_{z}$ can be interpreted as senders' noise contained in the signals, which cannot be reduced by paying attention to the signals. One implicit assumption is that agents cannot directly observe the fundamental and can obtain information only through analyzing the noisy signals about it.

Following Sims (2003), we assume that agents have a finite capacity to process available information, and that the reduction in uncertainty about the true signals is limited by finite entropy. Therefore, agents can only observe the noisy signals:

$$
\hat{s}_{i}=\left(\begin{array}{c}
\hat{x}_{i} \\
\hat{z}_{i}
\end{array}\right)=\left(\begin{array}{c}
x_{i} \\
z
\end{array}\right)+\left(\begin{array}{c}
\xi_{x_{i}} \\
\xi_{z_{i}}
\end{array}\right),
$$

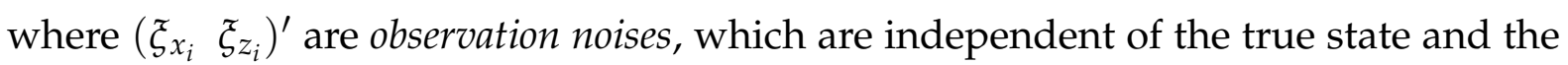
sender noises, and are independently and identically distributed across agents. The presence of observation noises reflects the finite information processing capacity. Its co-variance matrix is given by

$$
\Lambda=\left(\begin{array}{cc}
\omega_{x}^{2} & 0 \\
0 & \omega_{z}^{2}
\end{array}\right)
$$

where $\omega_{x}^{2}$ and $\omega_{z}^{2}$ are variances in the observation noises for private and public signals, respectively. Because the observation noises are idiosyncratic, noisy observation of the public signal, $\hat{z}_{i}$, is imperfectly correlated across the agents, whereas the observation of the private signal, $\hat{x}_{i}$, remains independent.

We define the posterior covariance matrix of $s_{i}$ with $\Psi \equiv \operatorname{Var}\left(s_{i} \mid \hat{s}_{i}\right)$, which can be determined using the following Gaussian updating formula,

$$
\Psi=\Sigma-\Sigma(\Sigma+\Lambda)^{-1} \Sigma \text { or } \Psi^{-1}=\Sigma^{-1}+\Lambda^{-1} .
$$

We assume that each agent in this economy possesses a limited amount of capacity to process information. Specifically, each agent is assumed to face the following information-processing constraint:

$$
\frac{1}{2} \ln \left(\frac{|\Sigma|}{|\Psi|}\right) \leq \kappa
$$

where $|\Sigma|$ and $|\Psi|$ denote the determinant of the prior covariance matrix of $s_{i}$ and the corresponding posterior covariance matrix, respectively, and $\kappa$ is positive and denote 
the individual's finite channel capacity. This constraint implies that the reduction in the uncertainty about the state gained from observing new signals is bounded from above by a finite capacity.

Given the specifications of (4), (6) and (7), the information processing constraint (8) can be rewritten as

$$
\underbrace{\frac{1}{2} \ln \left(\frac{\omega_{x}^{2}+\left(\sigma_{x}^{2}+\sigma^{2}\right)\left(1-\rho^{2}\right)}{\omega_{x}^{2}}\right)}_{\hat{\kappa}_{x}}+\underbrace{\frac{1}{2} \ln \left(\frac{\omega_{z}^{2}+\left(\sigma_{z}^{2}+\sigma^{2}\right)\left(1-\rho^{2}\right)}{\omega_{z}^{2}}\right)}_{\hat{\kappa}_{z}} \leq \hat{\kappa},
$$

where $\rho$ is the prior correlation across signals and $\hat{\kappa}$ is the effective capacity. They are defined by,

$$
\rho \equiv \operatorname{Corr}\left(x_{i}, z\right)=\sqrt{\frac{\sigma^{2} \sigma^{2}}{\left(\sigma_{x}^{2}+\sigma^{2}\right)\left(\sigma_{z}^{2}+\sigma^{2}\right)}}, \quad \hat{\kappa} \equiv \frac{1}{2} \ln \left(\exp (2 \kappa)-\rho^{2}(\exp (2 \kappa)-1)\right) .
$$

Effective capacity is the amount of capacity used to reduce observation noises while a certain amount of capacity must be "wasted" to learn the correlated part of the two signals twice. Intuitively, for a fixed amount of $\kappa$, the higher is the correlation between signals and the lower is the effective capacity, $\hat{\kappa}$. If the signals are independent, i.e., $\rho=0$, then $\kappa=\hat{\kappa}$.

The effective capacity spent on the private and public signals are denoted by $\hat{\kappa}_{x}$ and $\hat{\kappa}_{z}$, respectively. Naturally, we impose the following non-negativity restriction,

$$
\hat{\kappa}_{z} \geq 0, \quad \text { and } \quad \hat{\kappa}_{x} \geq 0 .
$$

The variances in observation noises can be recovered from equation (9) as follows:

$$
\omega_{x}^{2}=\frac{\left(\sigma_{x}^{2}+\sigma^{2}\right)\left(1-\rho^{2}\right)}{\exp \left(2 \hat{\kappa}_{x}\right)-1}, \quad \omega_{z}^{2}=\frac{\left(\sigma_{z}^{2}+\sigma^{2}\right)\left(1-\rho^{2}\right)}{\exp \left(2 \hat{\kappa}_{z}\right)-1} .
$$

If agents spend more effective capacity on observing a signal, then that signals' observation noise is smaller or it is clearer to the agents. In a limit case, agents possess an infinite amount of capacity and can therefore perfectly observe both signals, i.e., $\omega_{x}^{2}=0$ and $\omega_{z}^{2}=0$. To facilitate the characterization that follows, we define the relative accuracy of the public signal by

$$
\nabla \equiv \sqrt{\frac{\left(\sigma_{x}^{2}+\sigma^{2}\right)}{\left(\sigma_{z}^{2}+\sigma^{2}\right)}}
$$

and it can be readily verified that $\rho<\nabla<\frac{1}{\rho}$. 


\subsection{Equilibrium}

This model environment can be considered as a two-stage game. In the first stage, nature draws the underlying state and agents make decisions on their attention allocation by optimally splitting the effective capacity between the signals to be observed. In the second stage, agents observe the realized signals and then take action.

We focus on a linear symmetric equilibrium in which all agents follow the same strategy in attention allocation and adopt a linear strategy in actions. Because the attention allocation is determined in the first stage, the heterogeneity in signal observations in the second stage does not affect their decision. Once agents decide their attention allocation, the variances in observation noises are also determined. The action strategy $a_{i}$ in the second stage is linear in both the prior and observations on signals,

$$
a_{i}=\Pi_{\theta, i} \bar{\theta}+\Pi_{x, i} \hat{x}_{i}+\Pi_{z, i} \hat{z}_{i}
$$

where $\left(\Pi_{\theta, i}, \Pi_{x, i}, \Pi_{z, i}\right)$ are the weights assigned to the prior and observations.

We first solve the second-stage game, where the equilibrium remains unique. The solution to this game is the optimal weighting rule for any arbitrary allocation of attention in the first stage. Then, we solve for the optimal attention allocation in the first stage, taking the optimal weighting rule as given.

Given the linearity of the strategy and the normality of the information structure, we can show that an agent's action is a weighted average of the observations and their prior. That is,

$$
\Pi_{\theta, i}+\Pi_{x, i}+\Pi_{z, i}=1
$$

Individual $i$ 's payoff depends on the other agents' choices. Let the action strategy of the other agents be $\left(\hat{\kappa}_{x}, \hat{\kappa}_{z}, \Pi_{\theta}, \Pi_{x}, \Pi_{z}\right)$. The expected utility of individual $i, E\left[u_{i}\right]$, can be written as the sum of three components,

$$
\begin{aligned}
E\left[u_{i}\right]= & -\underbrace{\frac{1}{1-\alpha}\left(\Pi_{\theta, i}^{2} \frac{1}{\phi_{\theta}}+\Pi_{x, i}^{2} \frac{1}{\phi_{x, i}}+\Pi_{z, i}^{2} \frac{1}{\phi_{z, i}}\right)}_{\mathcal{L}^{+}}-\underbrace{\frac{\alpha}{1-\alpha}\left(\left(\Pi_{z, i}-\Pi_{z}\right)^{2} \sigma_{z}^{2}+\left(\Pi_{\theta, i}-\Pi_{\theta}\right)^{2} \sigma^{2}\right)}_{\mathcal{L}^{\ddagger}} \\
& +\mathcal{C},
\end{aligned}
$$

with

$$
\phi_{\theta}=\frac{1}{(1-\alpha) \sigma^{2}}, \quad \phi_{x, i}=\frac{1}{\sigma_{x}^{2}+\omega_{x, i}^{2}}, \quad \phi_{z, i}=\frac{1}{(1-\alpha) \sigma_{z}^{2}+\omega_{z, i}^{2}}
$$


and

$$
\mathcal{C}=\frac{-\alpha}{1-\alpha}\left[\left(\int a_{j}^{2} d j-\bar{a}^{2}\right)-\bar{L}\right] .
$$

The first component of the utility function, $-\mathcal{L}^{\dagger}$, is the quadratic loss of agent $i$, when everybody uses the same weights on their signals in action strategy. It shows that both sender and observation noises are of importance for the expected loss. The second component, $-\mathcal{L}^{\ddagger}$, is another possible source of expected loss for agent $i$ : utilizing a different action strategy from that used by the other agents. Obviously, when agent $i$ adopts the same strategy, $\mathcal{L} \ddagger$ becomes zero. Moreover, $\mathcal{C}$ is the effect of actions taken by other agents on agent $i$ and it cannot be affected by her choice. Note that $\omega_{x, i}^{2}$ and $\omega_{z, i}^{2}$ are the variances of agent $i$ 's observation noises on the private and public signals, respectively, which are given by (11), and $\bar{a}$ is the aggregate action.

\subsection{Characterization}

Agent $i$ maximizes $E\left[u_{i}\right]$ by choosing $\hat{\kappa}_{z, i}$ optimally. ${ }^{6}$ To analyze the equilibrium allocation of attention, we study agent $i$ 's best response allocation strategy. We begin by defining the relative marginal return of attention on the public signal, which turns out to be convenient in the analysis of the main mechanisms. That is,

$$
\gamma\left(\hat{\kappa}_{z, i}, \hat{\kappa}_{z}^{*}\right) \equiv \frac{\partial E\left[u_{i}\right] / \partial \hat{\kappa}_{z, i}}{\partial E\left[u_{i}\right] / \partial \hat{\kappa}_{x, i}}
$$

It is straightforward that $\gamma\left(\hat{\kappa}_{z, i}, \hat{\kappa}_{z}^{*}\right)$ measures the relative attractiveness of paying additional attention to the public signal, given others' attention allocation plan $\hat{\kappa}_{z}^{*}$. There are four forces that shape the attention allocation decision and thus affect $\gamma$. In the following sections, we fix the correlation between the two signals and discuss the role of the other three. We elaborate on the effect of the correlation in Section 4.2.

Lemma 1. $\gamma\left(\hat{\kappa}_{z, i}, \hat{\kappa}_{z}^{*}\right)$ decreases in $\hat{\kappa}_{z, i}$, increases in $\nabla$, and increases in $\alpha$.

First, the force of diminishing returns to attention takes hold. The more attention that is paid to the public signal, the less attractive it becomes. Second, agents prefer the signal with higher accuracy; that is, the higher its relative accuracy, the more attractive it is. Third, the coordination motive tilts agents' choice toward learning the public signal because they are rewarded in two ways when they spend more attention on the public signal: they are better informed about the underlying true state and their actions are better aligned. In other words, due to the coordination motive, the relative

\footnotetext{
${ }^{6}$ Note that the optimal weighting rule $\left(\Pi_{z, i}^{*} \Pi_{x, i}^{*} \Pi_{\theta, i}^{*}\right)$ in the second stage is uniquely determined by the attention allocation plan $\left(\hat{\kappa}_{z, i}^{*}, \hat{\kappa}_{x, i}^{*}\right)$.
} 
attractiveness of the public signal is magnified. The first part of Lemma 1 also implies that agent $i$ increases her attention on the public signal if and only if $\gamma\left(\hat{\kappa}_{z, i}, \hat{\kappa}_{z}^{*}\right)>1$, and decreases her attention if and only if $\gamma\left(\hat{\kappa}_{z, i}, \hat{\kappa}_{z}^{*}\right)<1$.

In symmetric equilibrium, we impose the condition that $\hat{\kappa}_{z, i}=\hat{\kappa}_{z}^{*}$, and three situations can arise. First, agents spend all of their attention on the public signal, where $\gamma\left(\hat{\kappa}_{z}^{*}, \hat{\kappa}_{z}^{*}\right)>1$ and $\hat{\kappa}_{z}^{*}=\hat{\kappa}$. Second, they spend all of their attention on the private signal, where $\gamma\left(\hat{\kappa}_{z}^{*}, \hat{\kappa}_{z}^{*}\right)<1$ and $\hat{\kappa}_{z}^{*}=0$. Third, they split their attention between both signals, where $\gamma\left(\hat{\kappa}_{z}^{*}, \hat{\kappa}_{z}^{*}\right)=1$ and $\hat{\kappa}_{z}^{*} \in[0, \hat{\kappa}]$. The following proposition offers the complete equilibrium characterization.

Proposition 1. In linear symmetric equilibria, agents adopt the same attention allocation strategy, where the equilibrium attention allocation is such that

$$
\begin{aligned}
& \hat{\kappa}_{z}^{*}= \begin{cases}0 & \text { if } \nabla \leq \nabla_{0} \\
\tilde{\hat{\kappa}}_{z} & \text { if } \nabla \in\left(\min \left\{\nabla_{0}, \nabla_{1}\right\}, \max \left\{\nabla_{0}, \nabla_{1}\right\}\right) \\
\hat{\kappa} & \text { if } \nabla \geq \nabla_{1}\end{cases} \\
& \hat{\kappa}_{x}^{*}=\hat{\kappa}-\hat{\kappa}_{z}^{*}
\end{aligned}
$$

where

$$
\tilde{\hat{\kappa}}_{z}=\frac{1}{2} \hat{\kappa}+\ln \left(\frac{(1-\alpha)(1-\rho \nabla)+(\nabla-\rho) \exp (\hat{\kappa})-\left(1-\rho^{2}\right)}{(1-\alpha)(1-\rho \nabla) \exp (\hat{\kappa})+(\nabla-\rho)-\nabla\left(1-\rho^{2}\right)}\right)
$$

and

$$
\nabla_{0}=\frac{\exp (\hat{\kappa}) \rho+1}{\exp (\hat{\kappa})+\rho}, \quad \nabla_{1}=\frac{(1-\alpha)(\exp (2 \hat{\kappa})-1)+\left(1-\rho^{2}\right)}{(1-\alpha)(\exp (2 \hat{\kappa})-1) \rho+\exp (\hat{\kappa})\left(1-\rho^{2}\right)}
$$

There exist multiple equilibria, i.e., $\hat{\kappa}_{z}^{*}=\left\{0, \tilde{\hat{\kappa}}_{z}, \hat{\kappa}\right\}$, if and only if

$$
\nabla_{1}<\nabla<\nabla_{0} \quad \text { and } \quad \alpha \geq 1-\frac{\rho}{\exp (\hat{\kappa})}
$$

otherwise, the equilibrium attention allocation is unique.

When the relative accuracy is extreme, agents find it optimal to focus on only one of the signals; that is, for a fixed amount of capacity $\hat{\kappa}$ and a coordination motive $\alpha$, if the relative accuracy is sufficiently high, i.e., $\max \left\{\nabla_{1}, \nabla_{0}\right\} \leq \nabla$, then agents choose to only observe the public signal. If the relative accuracy is sufficiently low, i.e., $\nabla \leq$ $\min \left\{\nabla_{1}, \nabla_{0}\right\}$, then agents choose to observe the private signal only.

When the relative accuracy is not too extreme, this model can admit either multiple 


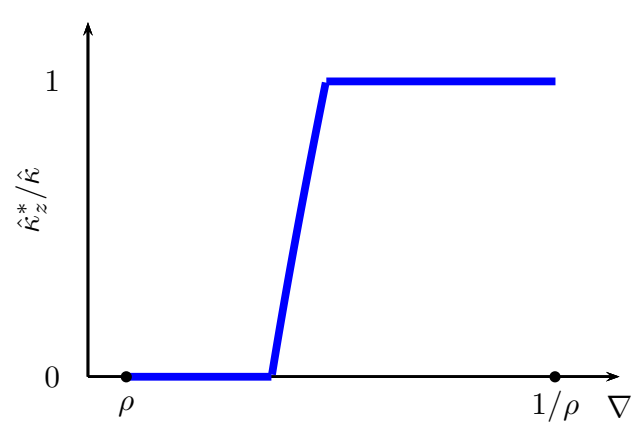

(a) $\alpha<1-\rho / \exp (\hat{\kappa})$.

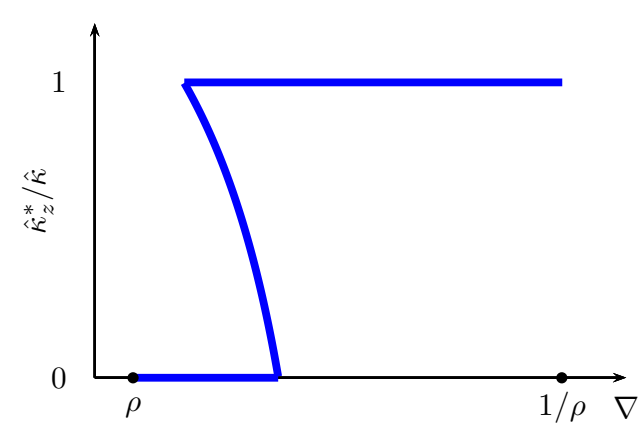

(b) $\alpha \geq 1-\rho / \exp (\hat{\kappa})$.

Figure 1. The equilibrium uniqueness and multiplicity.

equilibria or a unique equilibrium. ${ }^{7}$ See Figure 1 . Multiple equilibria can arise when the coordination motive (or correlation) is sufficiently high and/or the total amount capacity is relatively low. First, the relative accuracy cannot be too extreme for the existence of multiple equilibria. When everybody focuses on the lower quality signal, agent $i$ finds that the benefit of deviating and instead focusing on the relatively more precise signal is dominated by the cost of adopting a different strategy from other agents. Second, the coordination motive must be sufficiently large, such that when the strategic concern is strong, agents are more severely punished for deviating from the strategy adopted by other agents and therefore have less incentive to do so. Third, if the amount of capacity available is too large, then it is too costly for agent $i$ to follow the others' strategy, conditional on the rest of the population coordinating on a "wrong" choice. In contrast, there is only a unique equilibrium if the coordination motive (or correlation) is not sufficiently strong or capacity is large.

In both cases, a symmetric equilibrium is formed if all agents choose the global minimizer of $\mathcal{L}^{\dagger}$, because both $\mathcal{L}^{\dagger}$ and $\mathcal{L}^{\ddagger}$ (defined in equation (14)) achieve global minimization and no individual has an incentive to deviate from it. We label it strategic utility maximizing equilibrium, because it generates the maximum of strategic utility, which is defined by $E\left[u_{i}^{s}\right] \equiv-\mathcal{L}^{\dagger}-\mathcal{L}^{\ddagger}$; that is the component, on which the choice of agents has an influence.

\section{Attention Allocation}

In this section, we analyze the comparative statics of the equilibrium attention allocation, not only because the issue of attention allocation itself is interesting but also because it provides building blocks for our examination of social welfare in Section 5, and policy issues in Section 6. We also highlight the role that the correlation between

\footnotetext{
${ }^{7}$ Technically, $\mathcal{L}^{\dagger}$ can be either quasi-concave or quasi-convex in $\hat{\kappa}_{z, i}$. The equilibrium is unique if and only if it is quasi-concave. In this model, the entropy is not a convex function of signal precision and that is why multiple equilibria can possibly emerge in this model. In contrast, with the costly acquisition approach, the cost function of noise reduction is usually assumed to be convex.
} 
the public and private signals plays in attention allocation, as it is absent in most of the previous literature.

\subsection{Non-monotonic Attention Allocation}

For any relative accuracy and coordination motive, when the capacity is sufficiently large, the effect of diminishing returns eventually dominates, which leads to a diversified attention allocation. Because both signals can be extremely clear, the coordination motive and relative accuracy do not affect the attention allocation, with agents simply splitting a large amount of attention evenly between the two signals.

Proposition 2. The share of effective capacity devoted to the public signal, $\hat{\kappa}_{z}^{*} / \hat{\kappa}$, converges to $1 / 2$, when capacity is sufficiently high.

When the capacity is not large enough, the three forces characterized in Lemma 1 are intertwined and affect how the equilibrium attention allocation responds to an increase in capacity.

Proposition 3. If the public signal is less accurate than the private signal, agents specialize in learning the latter, when the capacity is low. When the capacity increases, the share of effective capacity devoted to the public signal is monotonically increasing, if the private signal is very precise or the coordination motive is not so strong; otherwise, it is hump-shaped.

In the proof of the proposition, we offer a complete characterization of this comparative statics. If the private signal is sufficiently accurate or the coordination motive is sufficiently low, it is never worthwhile to only observe the public signal, despite the effect of the coordination motive. The key trade-off here is between the effects of diminishing returns and relative accuracy, with the former eventually dominating the latter when the capacity is higher. Let $\hat{\kappa}_{0}$ be the threshold value of $\hat{\kappa}$, at which agents are indifferent about specialization in the private signal or diversification. In this case, when the capacity is higher than $\hat{\kappa}_{0}$, the share of attention devoted to the public signal monotonically increases in $\hat{\kappa}$. See Figure $2(a)$.

If the coordination motive is strong, its effect manifests in the non-monotonicity of the share of attention spent on the public signal. See Figure 2(b). When there is an increase in capacity, both the diminishing returns and the coordination motive have larger effects, and both forces tilt the choice of attention allocation toward the public signal. Thus, there is a sharp increase in $\hat{\kappa}_{z}^{*} / \hat{\kappa}$. However, when agents allocate a predominant share of their attention to the public signal, the mechanism of diminishing returns to attention takes stronger effect and pushes agents to diversify. The effect of the coordination motive is eventually dominated and therefore, $\hat{\kappa}_{z}^{*} / \hat{\kappa}$ decreases in $\hat{\kappa}$. 


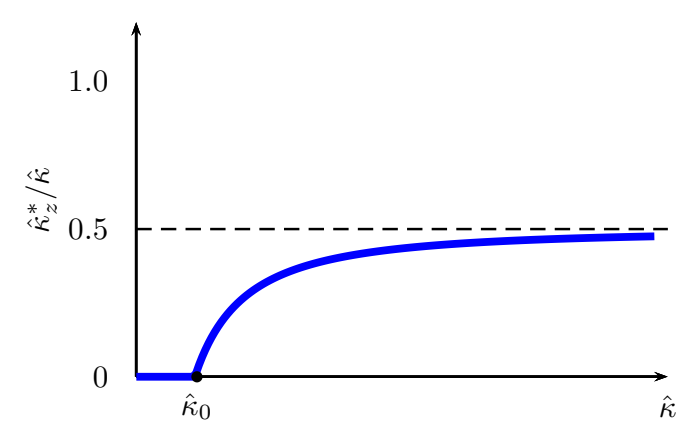

(a) Low $\nabla$ and Low $\alpha$

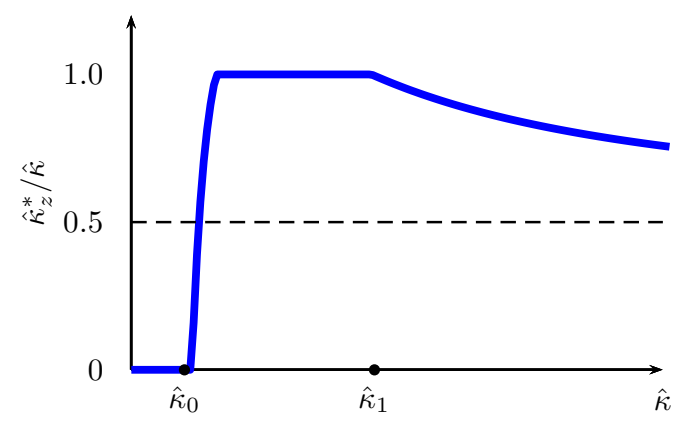

(c) Low $\nabla$ and High $\alpha$

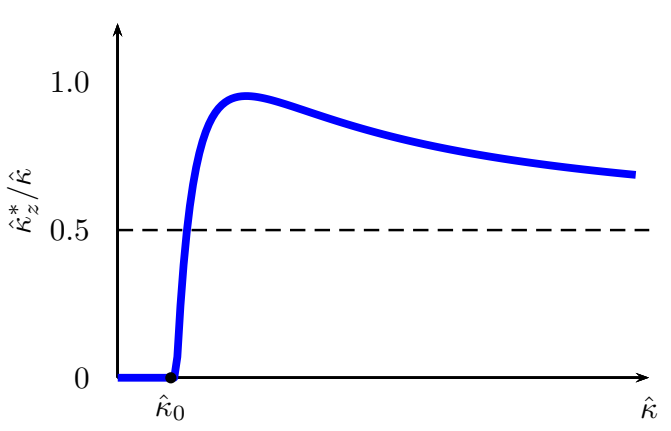

(b) Very Low $\nabla$ and High $\alpha$

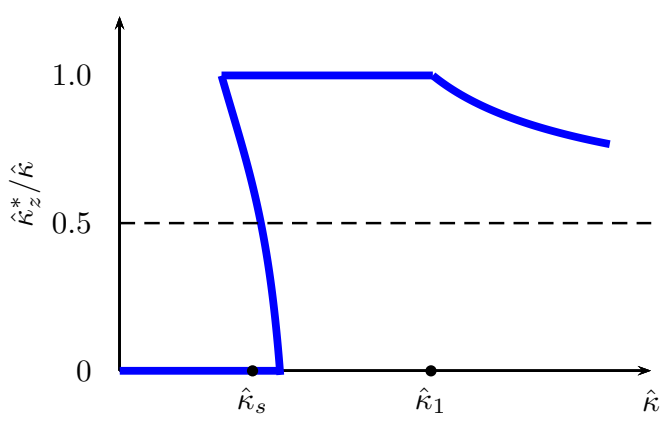

(d) Low $\nabla$ and Very High $\alpha$

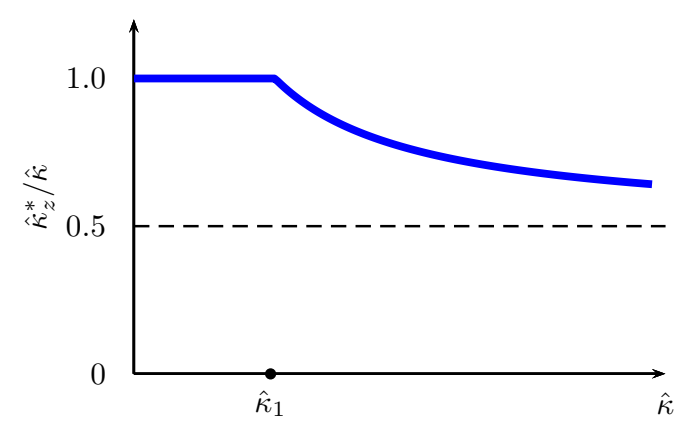

(e) High $\nabla$

Figure 2. The equilibrium share of attention allocated to the public signal is either monotonic or humpshaped in effective capacity.

If the precision of the private signal is close to that of the public one, the effect of the strong coordination motive can be so prominent that the share of attention on the public signal can reach $100 \%$. See Figure 2(c). It is interesting to observe that in this case, agents' attention fans out, contracts inward, then fans out again. The number of signals that agents pick up does not monotonically increase in capacity.

Notably, $\hat{\kappa}_{z}^{*} / \hat{\kappa}$ being hump-shaped implies that, the absolute amount of attention paid to the more precise private signal can decrease (even to zero) when the total amount of capacity increases, as the result of a strong coordination motive. We formalize this mechanism with "attention misallocation," and demonstrate further how this mechanism critically affects the social welfare of this economy in Section 5.

Definition 1. (Attention misallocation) Let $j$ be the relatively more precise signal, i.e., $\sigma_{j}=$ $\min \left\{\sigma_{x}, \sigma_{z}\right\}$ and $j \in\{x, z\}$. Attention misallocation arises, if the absolute amount of attention 
paid to the signal $j$ in equilibrium decreases in response to an increase in total capacity, i.e., $\mathrm{d} \hat{\kappa}_{j}^{*} / \mathrm{d} \hat{\kappa}<0$.

Lemma 2. When the private signal is more precise than the public signal, the absolute amount of effective capacity allocated to observing the private signal can even decrease in the total amount of capacity, on the condition that $2 \alpha+\rho>1$. Specifically, $\mathrm{d} \hat{\kappa}_{x}^{*} / \mathrm{d} \hat{\kappa}<0$.

When the coordination motive is very strong, all three equilibria can exist in the intermediate range of capacity. See Figure 2(d). Intuitively, this is the case in which none of the effects of relative accuracy, diminishing returns or coordination motive dominate the other two. Once other agents adopt one of the strategies, it is costly to deviate because the coordination motive is very high. Note that, in this case, the diversification equilibrium can never be the strategic utility maximizing equilibrium. ${ }^{8}$ Therefore, if we focus on the strategic utility maximizing equilibrium, agents can shift their focus entirely from the private to the public signal when capacity crosses a cutoff value of $\hat{\kappa}_{s}$. The key trade-off here is between taking advantage of high accuracy and the desire for coordination.

In contrast, if the public signal is relatively more accurate, agents specialize in learning the public signal to take advantage of both higher accuracy and better coordination when the capacity is lower than $\hat{\kappa}_{1}$, i.e., the threshold value at which agents are indifferent between specialization or diversification. They eventually diversify, due to the effect of diminishing returns, and the equilibrium share of attention devoted to the public signal decreases monotonically. See Figure 2(e).

\subsection{The Role of Correlation}

In this section, we turn to the role of correlation. The indirect effect of a change in correlation is straightforward. For any capacity $\kappa$, a higher correlation reduces the effective capacity available to agents, $\mathrm{d} \hat{\kappa} / \mathrm{d} \rho<0$. Intuitively, because the two signals are correlated, observing both of them costs agents some capacity to learn the correlated part twice. The direct effect is characterized in the following proposition.

Proposition 4. For any effective capacity, a higher correlation dampens the effect of diminishing returns to attention and amplifies the effect of the coordination motive. Specifically, (i) both $\hat{\kappa}_{0}$ and $\hat{\kappa}_{1}$ increase in $\rho$; (ii) $\hat{\kappa}_{s}$ decreases in $\rho$.

First, for any amount of effective capacity available to agents, the observation noises are reduced more effectively when the correlation is higher. See equation (11).

\footnotetext{
${ }^{8}$ When there exist multiple equilibria, $\mathcal{L}^{\dagger}$ is quasi-convex and the diversification allocation leads to a local minimum of $E\left[u_{i}^{s}\right]$.
} 
Because the two signals are correlated, knowing one of the signals helps reduce the other's observation noise. Therefore, agents have a stronger incentive to focus on one of the signals and the effect of diminishing returns is mitigated.

To demonstrate this, consider the case where only unique equilibrium exists for any capacity. ${ }^{9}$ Due to the effect of diminishing returns, agents switch from specialization to diversification when the effective capacity is higher than $\hat{\kappa}_{0}$ for the case of $\nabla<1$ and $\hat{\kappa}_{1}$ for the case of $\nabla>1$. When the correlation is higher, both cutoff values increase. That is, agents find it worthwhile to diversify only when the effective capacity is at a higher level. In this respect, the effect of an increase in the correlation differs from that of a rise in the coordination motive $\alpha$, which raises $\hat{\kappa}_{1}$ and lowers $\hat{\kappa}_{0}$.

Second, the correlation across signals also amplifies the effect of the coordination motive. Consider the case where the private signal is more precise. The rise in correlation entails a change in the trade-off between relative accuracy and coordination motive. In such a case, if agents spend more of their attention on the public signal, they estimate the underlying state less accurately but they can better align their actions. When the correlation between the private and public signals is higher, the two signals become more "substitutable," in terms of estimating the fundamental. Therefore, agents incur less welfare loss when they spend capacity on the less accurate public signal and they favor the public signal even more.

There are three ways to see the effect of this mechanism. First, as shown in Proposition 1 , for any effective capacity level $\hat{\kappa}$, and relative accuracy $\nabla \in\left[\nabla_{1}, \nabla_{0}\right]$, multiple equilibria emerge in this model when either $\alpha$ or $\rho$ is sufficiently large. Second, when we consider the strategic utility maximizing equilibrium in this case, agents shift their focus from the private to the public signal at $\hat{\kappa}=\hat{\kappa}_{s}$. We observe that $\hat{\kappa}_{s}$ decreases in both $\rho$ and $\alpha$. Third, in Lemma 2, we show that, on the condition that either $\alpha$ or $\rho$ is sufficiently high, attention misallocation can arise.

\section{5. "Too Much of a Good Thing": Social Welfare Analysis}

Social welfare is the average distance of individual actions in society from the fundamental. Agents benefit more from predicting the average opinion than other individuals, but it is a zero-sum game at the society level. In other words, the coordination motive only affects individual welfare and disappears at the society level. In this section, we analyze the comparative statics of social welfare by focusing on the strategic utility maximizing equilibrium.

The expected social welfare, $E\left[\mathcal{W}^{s}(\mathbf{a}, \theta)\right]$, is a weighted average of $E\left[u_{i}^{s}\right]$, which is the objective expected utility maximized by agents, and the spillover effect, which is

\footnotetext{
${ }^{9}$ As shown in Proposition 1, it is the case where $\alpha+\rho / \exp (\hat{\kappa})<1$.
} 
not considered by agents. The spillover receives a higher weight in social welfare if the coordination motive, $\alpha$, is stronger.

$$
\begin{aligned}
E\left[\mathcal{W}^{s}(\mathbf{a}, \theta)\right] & =-E\left[\int_{i}\left(a_{i}-\theta\right)^{2}\right] \\
& =(1-\alpha) \underbrace{\left[-\sigma^{2}\left(1+\frac{\phi_{x}}{\phi_{\theta}}+\frac{\phi_{z}}{\phi_{\theta}}\right)^{-1}\right]}_{E\left[u_{i}^{s}\right]}+\alpha \underbrace{\left[-\Pi_{\theta}^{2} \sigma^{2}-\Pi_{z}^{2} \sigma_{z}^{2}\right]}_{\text {Spillover }} .
\end{aligned}
$$

The unintended spillover effect arises from agents' desire to align their actions and the fact that they do not consider the effects that their choices have on others. Agents make use of the common prior and their correlated noisy observations on the public signal, when they forecast the actions of others and choose their own actions. As both the prior and the public signal are noisy, the actions taken by agents may be anchored around commonly known but imprecise information. Therefore, the spillover contributes negatively to social welfare and its magnitude is determined by how precise the signals are, i.e., $\sigma^{2}$ and $\sigma_{z}^{2}$, and how much agents rely on them, i.e., $\Pi_{\theta}$ and $\Pi_{z}$.

Proposition 5. (Social Welfare and Capacity) When the capacity to process information increases, social welfare can decrease. Specifically, there may exist $\hat{\kappa}_{a}<\hat{\kappa}_{b}$, such that

$$
E\left[\mathcal{W}^{s}\left(\hat{\kappa}_{a}\right)\right]>E\left[\mathcal{W}^{s}\left(\hat{\kappa}_{b}\right)\right]
$$

It is interesting to observe that a higher capacity to process information does not necessarily imply higher social welfare. On the one hand, $E\left[u_{i}^{s} \mid \hat{x}_{i}, \hat{z}_{i}\right]$, the part of welfare optimized by agents, always increases in capacity. On the other hand, the spillover can cause a decrease in social welfare when there is an increase in capacity. We know that spending more attention on the private signal reduces the spillover and enhances social welfare by lowering $\Pi_{z}$. However, recall the mechanism of attention misallocation shown in Lemma 2, agents may decrease the absolute amount of attention on the more accurate private signal when capacity increases, which results in a higher $\Pi_{z}$. Therefore, an increase in capacity can be detrimental to social welfare. In addition to this mechanism, holding constant $\hat{\kappa}_{x}^{*}$, a higher capacity implies that the amount of attention allocated to the public signal increases. Therefore, agents assign a larger weight to their observations on the public signal, $\hat{z}_{i}$, which is also socially costly. ${ }^{10}$ In short, a strong coordination motive or a high correlation between signals can distort

\footnotetext{
${ }^{10}$ We show that $\partial \Pi_{\theta} / \partial \hat{\kappa}_{x}^{*}=0$; that is, the increase in $\phi_{x}$ must equal the decrease in $\phi_{z}$ when $\hat{\kappa}_{x}^{*}$ is optimally chosen. $\partial \Pi_{\theta} / \partial \hat{\kappa}<0$, as holding $\hat{\kappa}_{x}^{*}$ constant, a higher capacity implies a higher $\phi_{z}$ and therefore, $\Pi_{\theta}$ must decrease. Intuitively, when the capacity is higher, agents rely more on their observation(s) and less on their prior knowledge.
} 


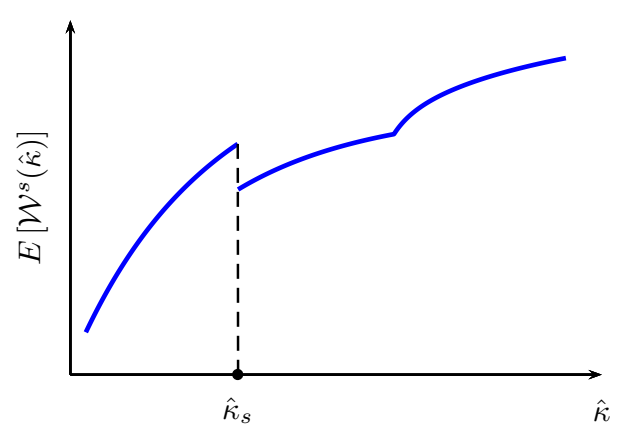

(a) $\alpha=0.7 ; \rho=0.8 ; \nabla=0.9135$

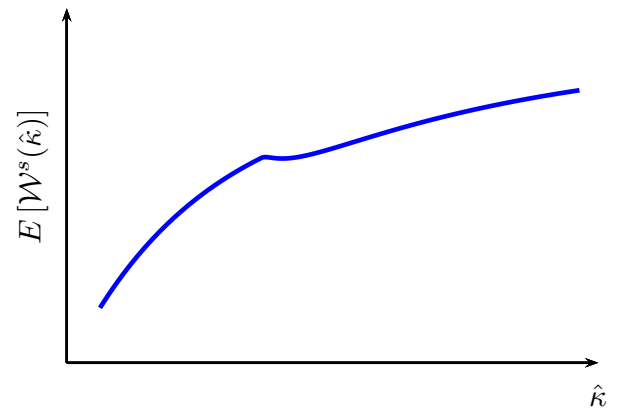

(b) $\alpha=0.7 ; \rho=0.8 ; \nabla=0.8971$

Figure 3. The non-monotonicity of social welfare.

the allocation of attention so much that the spillover increases quickly in response to a higher capacity, which results in a decrease in overall social welfare.

To demonstrate this mechanism, we choose a set of parameters with high coordination motive (or high correlation) and intermediate relative accuracy, such that agents switch their attention entirely from the private signal to public signal at $\hat{\kappa}_{s}$, in the strategic utility maximizing equilibrium. ${ }^{11}$ When $\hat{\kappa}=\hat{\kappa}_{s}$, agents are indifferent about only observing the private signal or the public signal; that is, $E\left[u_{i}^{s} \mid \hat{x}_{i}\right]=E\left[u_{i}^{s} \mid \hat{z}_{i}\right]$. In other words, $\phi_{x}=\phi_{z}$. For the same reason, $\Pi_{\theta}$ is the same in both cases. When $\hat{\kappa}$ increases from $\hat{\kappa}_{s}^{-}$to $\hat{\kappa}_{s}^{+}, \hat{\kappa}_{x}^{*}$ decreases from $\hat{\kappa}_{s}$ to 0 and $\Pi_{z}$ jumps from 0 to $1-\Pi_{\theta}$, so that there is a discontinuous decrease in social welfare. Because $E\left[\mathcal{W}^{s}\right]$ monotonically increases in $\hat{\kappa}$, when $\hat{\kappa}<\hat{\kappa}_{s}$, there must exist $\hat{\kappa}_{a}$ and $\hat{\kappa}_{b}$ such that $E\left[\mathcal{W}^{s}\left(\hat{\kappa}_{a}\right)\right]>E\left[\mathcal{W}^{s}\left(\hat{\kappa}_{b}\right)\right]$ and $\hat{\kappa}_{a}<\hat{\kappa}_{s}<\hat{\kappa}_{b}$. See Figure 3(a).

If the accuracy of the private signal is higher (or $\nabla$ is lower), the absolute amount of attention paid to the private signal decreases gradually and the weight assigned to the observation on the public signal also increases gradually. Therefore, social welfare may decrease continuously in capacity. See Figure 3(b). The following equation summarizes the key mechanisms discussed above, where the sign of $+(-)$ stands for a derivative being positive (negative).

$$
\frac{\mathrm{d} E\left[\mathcal{W}^{s}\right]}{\mathrm{d} \hat{\kappa}}=(1-\alpha) \underbrace{\frac{\mathrm{d} E\left[u_{i}^{s}\right]}{\mathrm{d} \hat{\kappa}}}_{+}+\alpha[\underbrace{\frac{\mathrm{d}\left(-\Pi_{\theta}^{2} \sigma^{2}\right)}{\mathrm{d} \hat{\kappa}}}_{+}+\underbrace{\frac{\partial\left(-\Pi_{z}^{2} \sigma_{z}^{2}\right)}{\partial \hat{\kappa}_{x}^{*}}}_{+} \underbrace{\frac{\mathrm{d} \hat{\kappa}_{x}^{*}}{\mathrm{~d} \hat{\kappa}}}_{+/-}+\underbrace{\frac{\partial\left(-\Pi_{z}^{2} \sigma_{z}^{2}\right)}{\partial \hat{\kappa}}}_{-}] .
$$

\footnotetext{
${ }^{11}$ This situation arises, when $\alpha>1-\rho$ and $\tilde{\nabla}<\nabla<1$, where $\tilde{\nabla}$ is defined in the proof of Proposition 3. Under this set of parameters, this model admits multiple equilibria and in this example, we focus on the change of social welfare in response to an change in capacity in a strategic utility maximizing equilibrium. However, this result does not rely on this particular case. In fact, the proof of Proposition 6 also implies Proposition 5. To establish Proposition 6, we focus on cases in which only a unique equilibrium exists.
} 


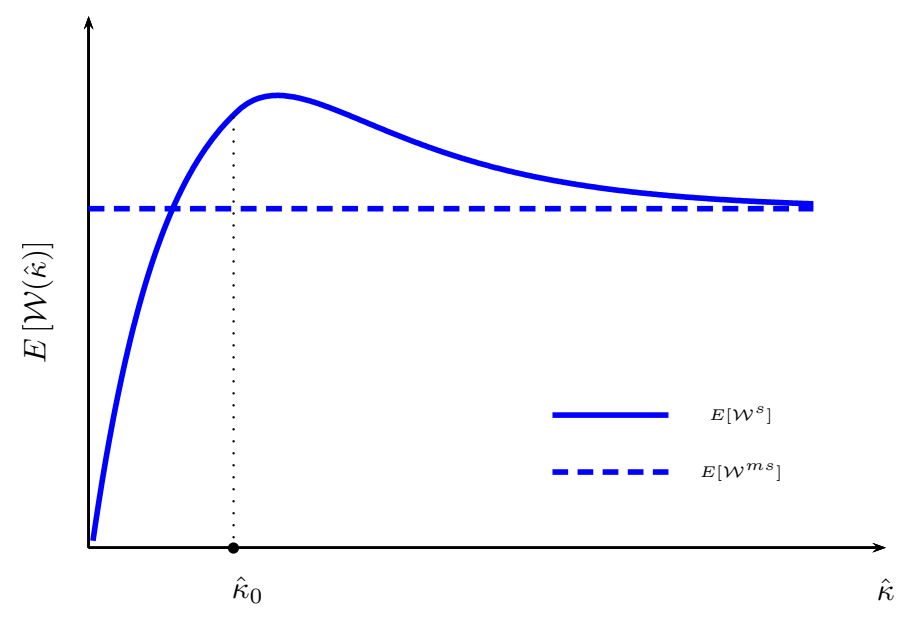

Figure 4. Finite vs. infinite capacity.

Proposition 6. (Too Much Capacity) Social welfare can be higher when agents are endowed with a finite amount of capacity to process information than when they have an infinite amount of capacity. Specifically, there is a finite $\hat{\kappa}^{\prime}$, such that

$$
E\left[\mathcal{W}^{s}\left(\hat{\kappa}^{\prime}\right)\right]>\lim _{\hat{\kappa} \rightarrow+\infty} E\left[\mathcal{W}^{s}(\hat{\kappa})\right] \equiv E\left[\mathcal{W}^{m s}\right]
$$

This result is striking. When agents possess an infinite amount of capacity to process information, they can perfectly observe both signals. In this case, the model is identical to the Morris-Shin model, in which the social inefficiency is well understood, i.e., agents overreact to the public signal. Specifically, the weight agents assign to the public signal in their action is higher than that in their posterior belief, which is socially costly because the coordination motive driving the overreaction does not count in social welfare. Social welfare in the Morris-Shin model can be written as follows

$$
E\left[\mathcal{W}^{m s}\right]=-\left[\frac{\phi_{x}^{m s}+\phi_{z}^{m s}+\frac{1}{(1-\alpha)} \phi_{\theta}}{\left(\phi_{x}^{m s}+\phi_{z}^{m s}+\phi_{\theta}\right)^{2}}+\frac{\Pi_{z}^{m s} \frac{\alpha}{(1-\alpha)}}{\phi_{x}^{m s}+\phi_{z}^{m s}+\phi_{\theta}}\right] \frac{1}{\sigma^{4}}
$$

where variables with superscript $m s$ are counterparts in the Morris-Shin model.

In our case, capacity-limited agents cannot clearly observe signals; thus, their estimation of the underlying state is less accurate than that when they have an infinite amount of capacity. However, agents may endogenously choose to spend very little attention on observing the public signal, as a result, the total amount of noise in the observation, $\hat{z}_{i}$, becomes very large. Therefore, they rely on it much less when they take actions; that is, the weight that it is assigned, $\Pi_{z}$, can be lower than $\Pi_{z}^{m s}$. A lower level of capacity can actually be welfare enhancing, because it does, to some extent, correct the inefficient use of public information. If the second effect dominates the first, social welfare can be higher than that in the Morris-Shin model. See Figure 4. 
We demonstrate the two opposing effects with the following simple case. Let the total amount of capacity in our model be $\hat{\kappa}_{0}$. We choose a set of parameter such that agents are indifferent between specialization in the private signal or diversification; that is, they endogenously ignore the public signal, or, $\phi_{z}=0 .{ }^{12}$ We write social welfare as follows

$$
E\left[\mathcal{W}^{s}\right]=-\left[\frac{\phi_{x}+\frac{1}{(1-\alpha)} \phi_{\theta}}{\left(\phi_{x}+\phi_{\theta}\right)^{2}}\right] \frac{1}{\sigma^{4}} .
$$

Given the finite capacity, agents cannot perfectly observe the private signal and thus the precision of observation of the private signal is smaller; that is, $\phi_{x}<\phi_{x}^{m s}$. Moreover, in the Morris-Shin model, the public signal is also informative and enhances the estimation of the underlying state, which results in $\phi_{x}<\phi_{x}^{m s}+\phi_{z}^{m s}$. Intuitively, agents with finite capacity are always worse off in terms of estimating the underlying state. It always holds that

$$
-\left[\frac{\phi_{x}+\frac{1}{(1-\alpha)} \phi_{\theta}}{\left(\phi_{x}+\phi_{\theta}\right)^{2}}\right] \frac{1}{\sigma^{4}}<-\left[\frac{\phi_{x}^{m s}+\phi_{z}^{m s}+\frac{1}{(1-\alpha)} \phi_{\theta}}{\left(\phi_{x}^{m s}+\phi_{z}^{m s}+\phi_{\theta}\right)^{2}}\right] \frac{1}{\sigma^{4}} .
$$

The second term in $E\left[\mathcal{W}^{m s}\right]$ shows the additional welfare loss caused by overusing the public signal in the Morris-Shin model. There is no overuse of the public signal in this particular finite-capacity case in that $\Pi_{z}=0$, because $\phi_{z}=0$. The socially costly overreaction to the public signal does not exist in this case

$$
0>-\left[\frac{\Pi_{z}^{m s} \frac{\alpha}{(1-\alpha)}}{\phi_{x}^{m s}+\phi_{z}^{m s}+\phi_{\theta}}\right] \frac{1}{\sigma^{4}} .
$$

When social inefficiency is high in the Morris-Shin model, the welfare loss due to "overreaction" to the public signal can be so large that the gain from a better estimation of the fundamental is dominated.

Given that the capacity can be "too much," is it possible for agents to voluntarily burn some capacity to achieve higher welfare? The answer is no. That is because, if everyone else collectively discards some of their capacity, individual $i$ can increase her welfare by fully using all of her capacity to enhance the estimation of the fundamental in the first stage and adopting the same action strategy in the second stage to avoid being "punished" for using a different strategy. ${ }^{13}$

\footnotetext{
${ }^{12}$ Section 4 demonstrates that such a $\hat{\kappa}_{0}$ exists, unless both $\alpha+\rho>1$ and $\nabla>\tilde{\nabla}$ hold, where $\tilde{\nabla}$ is defined in the proof of Proposition 3.

${ }^{13}$ This argument can be formalized and its proof is available on request.
} 


\section{Policy Issues}

In previous sections, we have fully characterized the optimal attention allocation and explored its implications for social welfare via comparative statics. In this section, we discuss two welfare-related issues to shed light on how policy prescriptions in the literature can be amended, considering that agents are capacity-constrained.

\subsection{Generalization and Efficient Use of Information}

Angeletos and Pavan (2007) offer a flexible efficiency benchmark to assess the welfare properties of a general class of games where the social value of coordination may be higher or lower than the private one. In this section, we demonstrate that our key results on social welfare can continue to hold in that generalized environment. Moreover, we also explain how the equilibrium multiplicity generated by our model mechanism would affect policy prescriptions offered in their work.

To accommodate this analysis, we enrich the payoff structure in this model by following Angeletos and Pavan (2007), where the utility function is specified by a general linear quadratic function, $u=U\left(k, K, \sigma_{k}, \theta\right)$, where $k$ and $K$ are individual and aggregate actions, respectively; $\sigma_{k} \equiv \int_{i}(k-K)^{2} \mathrm{~d} i$ is the action dispersion. In fact, we can write the utility function as follows,

$$
\begin{aligned}
u \equiv & \underbrace{-\left(k_{i}-\theta\right)^{2}-r\left(k_{i}-K\right)^{2}+r \sigma_{k}^{2}}_{I} \\
& +\underbrace{\left(\frac{U_{\sigma \sigma}}{2}-r\right) \sigma_{k}^{2}}_{I I}+\underbrace{\left(\frac{U_{K K}}{2}+r\right) K^{2}}_{I I I}+\underbrace{\left(U_{k K}-2 r\right) k K}_{I V} \\
& +\underbrace{\left(\frac{U_{\theta \theta}}{2}+1\right) \theta^{2}}_{V}+\underbrace{\left(\frac{U_{k k}}{2}+1+r\right) k^{2}}_{V I}+\left(U_{k \theta}-2\right) k \theta+U_{K \theta} K \theta .
\end{aligned}
$$

where $r$ is a positive constant. Our model is a special case, where we have $U_{\sigma \sigma}=2 r$, $U_{K K}=-2 r, U_{k K}=2 r, U_{\theta \theta}=-2, U_{k k}=-2(1+r), U_{k \theta}=2$ and $U_{K \theta}=0 .{ }^{14}$ Part $I$ is the standard beauty contest utility specification, by letting $\alpha=\frac{r}{1-r}$.

The private value of coordination, or how much agents care about aligning their actions, is measured by $\alpha$. The socially optimal degree of coordination is represented by $\alpha^{*}$ and it is the weight that the social planner would assign to the aggregate action

\footnotetext{
${ }^{14}$ We restrict our attention to the case where there is no inefficiency under complete information. That is, we impose a restriction on $U_{k \theta}$ and $U_{K \theta}$, which is standard as in Angeletos and Pavan (2007).
} 
in its best response. In the general case, they can be characterized as follows,

$$
\alpha^{*}=1-\frac{U_{k k}+2 U_{k K}+U_{K K}}{U_{k k}+U_{\sigma \sigma}} \quad \alpha=1-\frac{U_{k k}+U_{k K}}{U_{k k}} .
$$

Note that the beauty contest game is a special case with $\alpha^{*}=0$ (i.e., the social planner does not value coordination) and with $\alpha>0$ (i.e., individuals care about aligning their actions). Efficient attention allocation and efficient use of information are derived by solving a social planner problem while respecting the information processing constraint (9).

First, in the benchmark case, we have demonstrated in Proposition 5 and Lemma 2 that social welfare may decrease in response to an increase in capacity due to attention misallocation, on condition that coordination motive and/or correlation between signals are sufficiently strong. Alternatively, this result can be interpreted as follows. The social planner does not value coordination among agents at all in the beauty contest case. When the private value of coordination perceived by the agents is high, the equilibrium allocation may deviate a lot from the social optimum. Therefore, a further increase in capacity can result in an even severer attention misallocation, which may cause a reduction in social welfare.

In this general case, this mechanism can still produce the same effect, that social welfare decreases in capacity, when there is a large enough discrepancy between the value of coordination perceived by individuals, $\alpha$, and the central planner, $\alpha^{*}$. To illustrate this point, consider the following scenario. Suppose the payoff function is parametrized such that $\alpha=\alpha^{*}$. A positive $U_{\sigma \sigma}$ implies that the action dispersion has positive externality on individuals' payoffs. An increase in $U_{\sigma \sigma}$ reduces the social value of coordination, $\alpha^{*}{ }^{15}$ Therefore, the central planner prefers a higher action dispersion and allocating even less attention to the public signal and more attention to the private signal. However, the equilibrium value of coordination is not affected by the change in $U_{\sigma \sigma}$. When $U_{\sigma \sigma}$ is large enough, the attention misallocation, due to the discrepancy in equilibrium and efficient degree of coordination, can intensify and cause a decrease in social welfare when capacity increases. ${ }^{16}$

Second, one of the key insights in Angeletos and Pavan (2007) with the exogenous information structure is that the equilibrium use of information is efficient if and only if the social and private values of coordination coincide. However, this result may not hold once we allow for an endogenous information structure. Further, in this model, even though attention allocation is efficient, it does not necessarily lead to an efficient use of information.

\footnotetext{
${ }^{15}$ Note that both $U_{k k}+2 U_{k K}+U_{K K}$ and $U_{k k}+U_{\sigma \sigma}$ are negative in this case.

${ }^{16}$ Similar arguments can be applied to other cases and detailed analysis is available on request.
} 
In contrast to Angeletos and Pavan (2007), we argue that even when the central planner corrects the coordination incentives of agents to the socially optimal level with a tax policy, the equilibrium attention allocation and the use of information may still be not socially optimal. The key to understanding this argument is to recall that multiple equilibria may arise. Consider the case where the socially optimal degree of coordination $\alpha^{*}$ is higher than the private value of coordination $\alpha$ and it is so high that there exist multiple solutions in the central planner's social welfare optimization problem. It is obvious that the central planner picks the solution that gives rise to the highest social welfare. In a decentralized economy, with a policy similar to that proposed in Angeletos and Pavan (2007), the central planner can incentivize agents to value the coordination as much as it does, but it is still undetermined on which equilibrium agents coordinate. The planner needs another set of tools that help direct agents to coordinate on the social welfare maximizing equilibrium.

Further, in this framework, the equilibrium use of information can still be inefficient, even though the equilibrium attention allocation is efficient. To see this, we assume that $\alpha>\alpha^{*}>0$. The social planner also values the coordination and may dictate that agents focus on the public signal and ignore the private one. Under the same conditions, individuals could also choose exactly the same attention allocation. In other words, the attention allocation is socially optimal. However, as the private and social values of coordination differ, in the second stage, individuals would assign a higher weight in their action strategy to the common prior than would the social planner. To understand this, recall the fact that the common prior serves a "free public signal," which does not require any attention, and observations on the public signal are imperfectly correlated across agents in this economy due to the idiosyncratic observation noises. Therefore, the equilibrium use of information is still not socially optimal.

\subsection{Transparency of Public Announcement}

This study adds another dimension to the debate about central bank transparency. Unlike the common presumption that higher transparency is always beneficial, Morris and Shin (2002) show that it may be detrimental to social welfare when the central bank delivers clearer public announcements. In their beauty contest model with an exogenous information structure, an increase in the precision of public information entails two opposing effects. On the one hand, it allows agents to better estimate the underlying fundamental. On the other hand, it also increases agents' reliance on the noisy public information in their actions, which is socially costly. Both effects are enlarged as the precision of public information increases. Morris and Shin (2002) show that social welfare is U-shaped, such that when the precision of the public signal is exceedingly low, the second effect dominates. Specifically, social welfare decreases in 


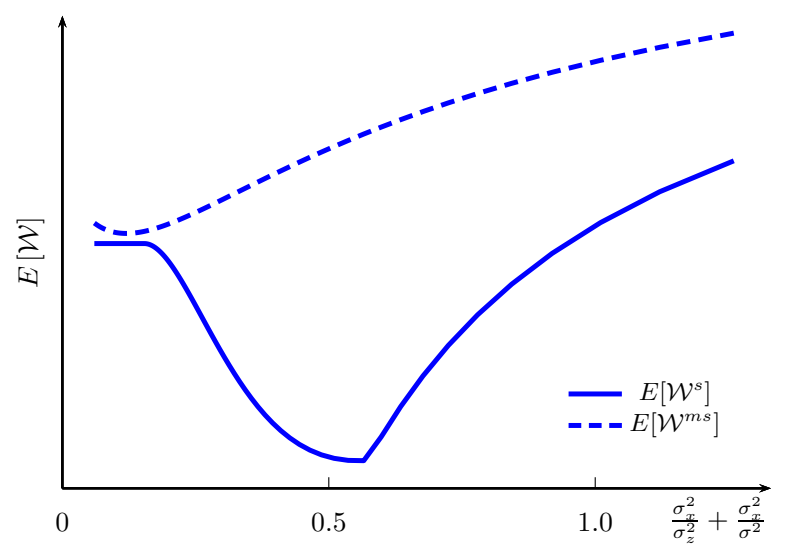

Figure 5. Central bank transparency and social welfare.

its precision if and only if

$$
\frac{\sigma_{x}^{2}}{\sigma_{z}^{2}}+\frac{\sigma_{x}^{2}}{\sigma^{2}}<(2 \alpha-1)(1-\alpha) .
$$

Therefore, it may be socially desirable to withhold public information.

One important critique of this argument is Svensson (2006), that questions its empirical relevancy and stresses that it can hold only when public information is implausibly imprecise. ${ }^{17}$ See the dashed line in Figure 5 where social welfare is plotted against the left side of equation (22), holding $\sigma_{x}^{2}$ and $\sigma^{2}$ fixed. ${ }^{18}$

However, we argue that the precision of the public signal needs not necessarily to be exceedingly low to generate a decline in social welfare, when we allow for endogenous attention allocation. When agents can decide to which information source they pay their attention, the precision of each signal that they observe becomes endogenous in that it not only depends on variances in sender noises, but also on those of observation noises, which are chosen by agents.

To illustrate this, we plot social welfare in our model with the solid line in Figure $5 .{ }^{19}$ When the precision of the public signal is very low, agents ignore it and focus on the private signal. Therefore, a marginal increase in the precision of the public signal does not affect social welfare. When the precision of the public signal is sufficiently high, agents diversify their attention. An increase in its precision leads to a higher reliance on the public signal in their action, as in Morris and Shin (2002). In addition,

\footnotetext{
${ }^{17}$ Even the maximum of the right side of (22) is a very small number, which implies that $\sigma_{z}^{2}$ must be sufficiently large for this condition to hold.

${ }^{18}$ In this numerical example, $\alpha=0.7$ and $\sigma_{x}=0.1 . \sigma$ is normalized to unit.

${ }^{19}$ In this numerical example, the capacity available to agents is $\kappa=3.2$ bits and all other parameters are the same as those for computing the counterpart in the Morris-Shin model. With this set of parameters, there is a unique equilibrium.
} 
agents also direct a larger proportion of their attention toward the public signal in response to a higher precision. This additional mechanism reinforces the previous one and both contribute to the decline in social welfare. As a result, social welfare still decreases, even when the precision of the public signal is reasonably large. ${ }^{20}$

\section{Alternative Information Structures}

Following Hellwig and Veldkamp (2009) and Myatt and Wallace (2012), we have made two implicit assumptions about the way in which agents learn in the benchmark model. First, agents cannot directly observe the fundamental, but can learn about it through analyzing noisy signals. In other words, agents can only obtain noisy observations about the underlying signals that are informative about the fundamental. ${ }^{21}$ That assumption is realistic in settings in which information acquisition takes the form of the assimilation of the available information and agents face a constraint to information transmission and comprehension. Second, the source of noisy information can be both public and private. ${ }^{22}$ That assumption captures the scenario that agents may have access to information sources of a different nature, i.e., economy-wide information that is commonly accessible and local information that is conditionally uncorrelated. $^{23}$ This set of assumptions, although common in the literature, is important for our results. The following discussion outlines how our results would change if we have allowed for alternative information structures.

\subsection{Observe the fundamental directly}

We first consider a case in which agents can pay attention to observe both the fundamental directly and a noisy public signal about the fundamental. Interestingly, this scenario is a special case in our benchmark model, in which the variance of the noise contained in the private signal $\sigma_{x}^{2}$ is 0 . In this case, the relative accuracy of the public

\footnotetext{
${ }^{20}$ When the precision of the public signal is high enough, agents pay all their attention to the public signal. In this situation, an increase in the precision of the public signal is always welfare-enhancing.

${ }^{21}$ The rational inattention model characterized by Myatt and Wallace (2012) allows agents to learn one noisy signal about the fundamental that contains exogenous sender's noise. They explicitly specify a cost function without studying the attention allocation problem, which is the focus of this paper.

${ }^{22}$ Hellwig and Veldkamp (2009) adopt the same set of assumptions, but they have a different specification for the way agents reduce the observation nosies. In their work, they allow agents to pay a fixed cost to obtain a noisy signal of the fundamental, which reduces the variance of the observation noise from infinity to zero. In contrast, we allow agents to choose the variance of the observation noises by spending capacity on those signals. Furthermore, the cost function of acquiring information is also different in their work.

${ }^{23}$ Private information can be interpreted alternatively. In an example of an island economy, islandlevel productivity can be a noisy signal about the aggregate productivity. Firms can obtain a noisy signal about local productivity by paying attention to it. The island-specific component, i.e., the difference between idiosyncratic island productivity and aggregate productivity, corresponds to the sender's noise in the private signal in our model. An increase in attention to the island-level productivity only reduces the observation noise.
} 
signal takes an extreme value $\nabla=\rho$. (Recall that $\nabla$ is always bounded by $\rho$ and $1 / \rho$.)

Two results arise from this extreme case. First, spending all of the endowed capacity on observing the fundamental directly is always an equilibrium and socially optimal. ${ }^{24}$ Intuitively, to estimate the fundamental $\theta$, spending capacity to observe $\theta$ directly is always more efficient than learning a noisy signal $z$ about it, in terms of reducing the observation noises. At the limit, when agents process an infinite amount of capacity, they only want to spend the capacity to observe $\theta$, in which case they can obtain the value of the fundamental. From the perspective of society, obtaining a clearer signal about the fundamental and discarding the public signal entirely is also the optimum, given that coordination is socially costly.

Second, agents may also coordinate on an inefficient equilibrium, in which everybody pays all his attention to the public signal and does not observe the fundamental at all. That case arises, only when the coordination motive is sufficiently strong, i.e., $\left(1+\sqrt{1-\rho^{2}}\right) / 2<\alpha<1$ and the total capacity is not too large or too small. ${ }^{25}$ The intuition is also not so different from the reason why multiple equilibria emerge in the benchmark case (discussed in Section 3.4). ${ }^{26}$

\subsection{Observe two public signals}

The assumption that the two information sources are of different publicity is important for our results. To highlight its effect, in this section we investigate an alternative setting where the two signals are both public but differ only in precision. ${ }^{27}$

Some results from this case are the same as those from our benchmark model. First, in this case, agents focus on the relatively more precise signal when the capacity available is rather small and diversify when it is very large. Second, it is also the case that multiple equilibria may emerge, when capacity is in the intermediate range. The intuitions gained from our benchmark model can be applied to explain these two results.

However, in this setting, it is not possible that agents pay full attention to the less accurate signal and ignore the relatively more precise one, unless multiple equilibria exist. In other words, the key mechanism of attention mis-allocation in our benchmark

\footnotetext{
${ }^{24}$ In other words, it holds that $\gamma(0,0)<1$ for any $\hat{\kappa}$.

${ }^{25}$ In this case, even though $\nabla$ takes the smallest possible value $\rho$, it is still possible that $\nabla_{1}<\nabla=\rho<$ $\nabla_{0}$. Recall that both $\nabla_{1}$ and $\nabla_{0}$ vary in $\hat{\kappa}$. In this case, $\hat{\nabla}$, that is, the minimum of $\nabla_{1}$, is smaller than $\rho$. See Figure 6(e) for an illustration of the attention allocation pattern, when $\nabla=\rho$.

${ }^{26}$ When the strategic concern is strong, agent $i$ is severely punished for deviating from the strategy of observing the public signal only, on the condition that everyone else adopts this strategy. To ensure that it is an equilibrium, the amount of capacity available cannot be too large; otherwise it is too costly for agent $i$ to follow this strategy, that is, spending capacity on observing fundamental directly is more efficient in terms of learning $\theta$. The amount of capacity cannot be too small either; otherwise the effect of relative accuracy dominates and agent $i$ chooses to deviate.

${ }^{27}$ We can provide a full characterization of this case upon request.
} 
model does not exist in this setting. Intuitively, if one of the signals is private and more precise and the other is public but less precise, it can be the case that agents may decrease their attention to, or totally ignore, the more precise private signal when the available capacity increases, because the desire of coordination dominates the effect of diminishing returns and relative accuracy. However, in the case of two public signals, that cannot be the case because both signals are public and can help coordinate agents' actions. Therefore, agents either pay full attention to the more precise public signal (when the effect of relative accuracy dominates), or divide their attention between the two public signals (when the effect of diminishing returns dominates).

\section{Conclusion}

There has been a recent surge of interest in modeling information acquisition and the endogenous information structure in macroeconomic environments. See Veldkamp (2011) for a textbook treatment on this topic and Hellwig, Kohls, and Veldkamp (2012) for an excellent review. However, fewer studies have touched on the welfare implications of information acquisition in this class of economies. This study focuses exclusively on a range of welfare issues in beauty contest models, in a context where agents are rationally inattentive and therefore optimally allocate a limited amount of attention between correlated private and public signals.

We fully characterize the sufficient and necessary conditions for the equilibrium uniqueness and multiplicity, and show that the attention allocation and the number of signals that agents decide to observe are not necessarily monotonic, in response to the increase in the capacity of processing information. Unlike the literature, we also highlight the role of the correlation between two signals, which critically affects the equilibrium uniqueness and multiplicity, along with the welfare properties in this model. Further, we show that in this setting, when capacity increases, the social welfare of this economy may not necessarily increase. In fact, it can decrease as a result of attention misallocation. Interestingly, social welfare can be even higher when agents possess a finite amount of capacity than when they have an infinite amount of capacity. 


\section{Appendix}

Proof of Lemma 1. First, we solve for the weighting rule adopted by all of the other agents, on the condition that their attention allocation is $\left(\hat{\kappa}_{x}^{*}, \hat{\kappa}_{z}^{*}\right)$ :

$$
\Pi_{x}^{*}=\frac{\phi_{x}^{*}}{\phi_{x}^{*}+\phi_{z}^{*}+\phi_{\theta}^{*}}, \quad \Pi_{z}^{*}=\frac{\phi_{z}^{*}}{\phi_{x}^{*}+\phi_{z}^{*}+\phi_{\theta}^{*}}, \quad \Pi_{\theta}^{*}=\frac{\phi_{\theta}^{*}}{\phi_{x}^{*}+\phi_{z}^{*}+\phi_{\theta}^{*}},
$$

where

$$
\phi_{x}^{*}=\frac{1}{\sigma_{x}^{2}+\left(\omega_{x}^{*}\right)^{2}}, \quad \phi_{z}^{*}=\frac{1}{(1-\alpha) \sigma_{z}^{2}+\left(\omega_{z}^{*}\right)^{2}}, \quad \phi_{\theta}^{*}=\frac{1}{(1-\alpha) \sigma^{2}}
$$

and

$$
\omega_{x}^{*}=\sqrt{\frac{\left(\sigma_{x}^{2}+\sigma^{2}\right)\left(1-\rho^{2}\right)}{\exp \left(2 \hat{\kappa}_{x}^{*}\right)-1}}, \quad \omega_{z}^{*}=\sqrt{\frac{\left(\sigma_{z}^{2}+\sigma^{2}\right)\left(1-\rho^{2}\right)}{\exp \left(2 \hat{\kappa}_{z}^{*}\right)-1}} .
$$

Second, we solve for the optimal action rule for agent $i$, i.e., $\left(\Pi_{z, i}^{*}, \Pi_{x, i}^{*}, \Pi_{\theta, i}^{*}\right)$, conditional on the others' allocation strategy $\left(\hat{\kappa}_{x}^{*}, \hat{\kappa}_{z}^{*}\right)$ and his own $\left(\hat{\kappa}_{x, i}, \hat{\kappa}_{z, i}\right)$. It is the solution to the following optimization problem,

$$
\max _{\Pi_{x, i}, \Pi_{z, i}, \Pi_{\theta, i}} E\left[u_{i}\right] \quad \text { s.t. }\left(\hat{\kappa}_{z, j}, \hat{\kappa}_{x, j}\right)=\left(\hat{\kappa}_{z}^{*}, \hat{\kappa}_{x}^{*}\right) \text { for all } j \neq i
$$

where $E\left[u_{i}\right]$ is given by equation (14). First order conditions imply that

$$
\begin{aligned}
\Pi_{x, i}^{*} & =\Pi_{x}^{*}+\frac{\left(c_{1} \Pi_{\theta}^{*}-c_{2} \Pi_{x}^{*}\right)\left(c_{1}+c_{5}+c_{3}+c_{4}\right)-\left(c_{1} \Pi_{\theta}^{*}-c_{3} \Pi_{z}^{*}\right)\left(c_{1}+c_{5}\right)}{\left(c_{1}+c_{5}+c_{2}\right)\left(c_{1}+c_{5}+c_{3}+c_{4}\right)-\left(c_{1}+c_{5}\right)^{2}}, \\
\Pi_{z, i}^{*} & =\Pi_{z}^{*}+\frac{\left(c_{1} \Pi_{\theta}^{*}-c_{3} \Pi_{z}^{*}\right)\left(c_{1}+c_{5}+c_{2}\right)-\left(c_{1} \Pi_{\theta}^{*}-c_{2} \Pi_{x}^{*}\right)\left(c_{1}+c_{5}\right)}{\left(c_{1}+c_{5}+c_{2}\right)\left(c_{1}+c_{5}+c_{3}+c_{4}\right)-\left(c_{1}+c_{5}\right)^{2}} .
\end{aligned}
$$

where

$$
\begin{array}{lll}
c_{1}=(1-\alpha) \sigma^{2}, & c_{2}=\sigma_{x}^{2}+\omega_{x, i}^{2}, & c_{3}=(1-\alpha) \sigma_{z}^{2}+\omega_{z, i}^{2}, \\
c_{4}=\alpha \sigma_{z}^{2}, & c_{5}=\alpha \sigma^{2} . &
\end{array}
$$

Therefore, the relative marginal return of attention on the public signal $\gamma$ can be rewritten by replacing $\Pi_{x, i}^{*}$ and $\Pi_{z, i}^{*}$ with (23) and (24).

$$
\gamma=\left[\frac{C_{1} \exp \left(2 \hat{\kappa}_{z, i}\right)+C_{2} \exp (2 \hat{\kappa})}{C_{3} \exp \left(2 \hat{\kappa}_{z, i}\right)+C_{4}}\right]^{2} \frac{1}{\nabla^{2} \exp (2 \hat{\kappa})} .
$$


where

$$
\begin{aligned}
& C_{1}=\left[(1-\alpha) \Pi_{\theta}^{*}+(1-\alpha) \Pi_{z}^{*}+\Pi_{x}^{*}\right](1-\nabla \rho), \\
& C_{2}=\alpha\left(\frac{1}{\rho^{2}}-1\right) \Pi_{z}^{*}+\left[(1-\alpha) \Pi_{\theta}^{*}+(1-\alpha) \Pi_{z}^{*}+\Pi_{x}^{*}\right]\left(\frac{\nabla}{\rho}-1\right), \\
& C_{3}=\left[(1-\alpha) \Pi_{\theta}^{*}+(1-\alpha) \Pi_{z}^{*}+\Pi_{x}^{*}\right]\left(\frac{1}{\nabla \rho}-1\right), \\
& C_{4}=\alpha\left(\frac{1}{\nabla \rho}-\frac{\rho}{\nabla}\right) \Pi_{z}^{*}+\left[(1-\alpha) \Pi_{\theta}^{*}+(1-\alpha) \Pi_{z}^{*}+\Pi_{x}^{*}\right]\left(1-\frac{\rho}{\nabla}\right) .
\end{aligned}
$$

Therefore, we can show

$$
\frac{\partial \gamma\left(\hat{\kappa}_{z, i}, \hat{\kappa}_{z}^{*}\right)}{\partial \hat{\kappa}_{z, i}}<0, \quad \frac{\partial \gamma\left(\hat{\kappa}_{z, i}, \hat{\kappa}_{z}^{*}\right)}{\partial \nabla}>0, \quad \frac{\partial \gamma\left(\hat{\kappa}_{z, i}, \hat{\kappa}_{z}^{*}\right)}{\partial \alpha}>0 .
$$

Proof of Proposition 1. The first part of Lemma 1 implies that the best response of agent $i$ to the allocation strategy adopted by others is unique. Therefore, the allocation $\left(\hat{\kappa}_{x}, \hat{\kappa}_{z}\right)=(\hat{\kappa}, 0)$ constitutes a symmetric equilibrium if and only if $\gamma(0,0)<1$. That is,

$$
\nabla \leq \frac{\exp (\hat{\kappa}) \rho+1}{\exp (\hat{\kappa})+\rho} \equiv \nabla_{0} .
$$

Similarly, the allocation $\left(\hat{\kappa}_{x}, \hat{\kappa}_{z}\right)=(0, \hat{\kappa})$ constitutes a symmetric equilibrium if and only if $\gamma(\hat{\kappa}, \hat{\kappa})>1$. That is,

$$
\nabla \geq \frac{(1-\alpha)(\exp (2 \hat{\kappa})-1)+\left(1-\rho^{2}\right)}{(1-\alpha)(\exp (2 \hat{\kappa})-1) \rho+\exp (\hat{\kappa})\left(1-\rho^{2}\right)} \equiv \nabla_{1} .
$$

A symmetric equilibrium with diversification must be such that $\hat{\kappa}_{z}^{*} \in(0, \hat{\kappa})$ and it exists if and only if $\gamma\left(\hat{\kappa}_{z}^{*}, \hat{\kappa}_{z}^{*}\right)=1$ where we have

$$
\gamma\left(\hat{\kappa}_{z}^{*}, \hat{\kappa}_{z}^{*}\right)=\frac{\left(\left(\frac{\nabla}{\rho}-1\right)\left(\exp (2 \hat{\kappa})-\exp \left(2 \hat{\kappa}_{z}^{*}\right)\right)+\nabla\left(\frac{1}{\rho}-\rho\right) \exp \left(2 \hat{\kappa}_{z}^{*}\right)\right)^{2}}{\exp (2 \hat{\kappa})\left((1-\alpha)\left(\frac{1}{\rho}-\nabla\right)\left(\exp \left(2 \hat{\kappa}_{z}^{*}\right)-1\right)+\left(\frac{1}{\rho}-\rho\right)\right)^{2}} .
$$

Such an equilibrium arises if

$$
\nabla \in\left(\min \left\{\nabla_{0}, \nabla_{1}\right\}, \max \left\{\nabla_{0}, \nabla_{1}\right\}\right)
$$

The optimal allocation is given by (18). Obviously, the equilibrium must be unique, if $\nabla_{0}<\nabla_{1}$, which also implies $\alpha<1-\frac{\rho}{\exp (\hat{\kappa})}$. In other words, multiple equilibria emerge if and only if the condition (20) holds. 


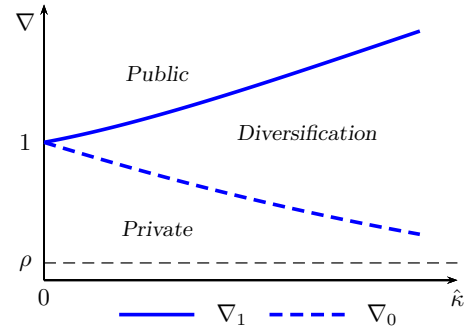

(a) $0<\alpha \leq(1-\rho) / 2$

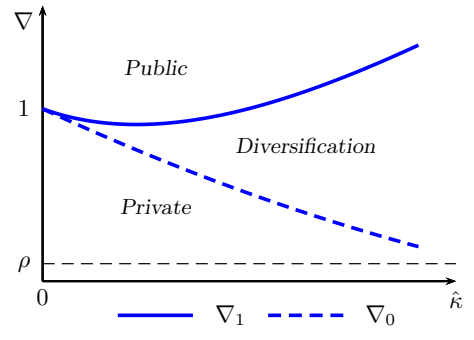

(b) $(1-\rho) / 2<\alpha \leq 1-\rho$

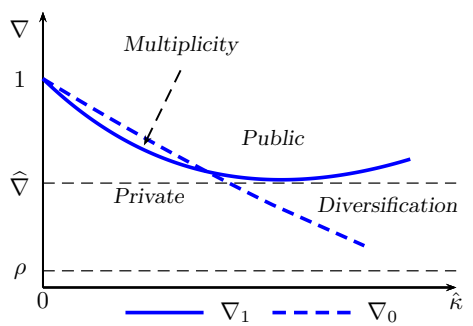

(c) $1-\rho<\alpha \leq 1-\rho^{2}$

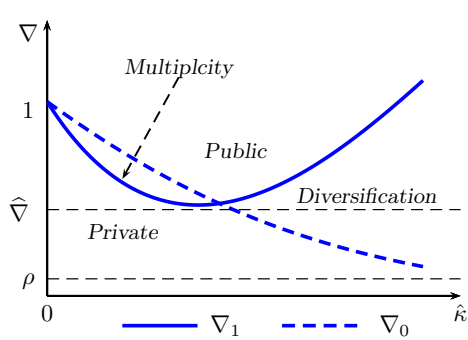

(d) $1-\rho^{2}<\alpha \leq \frac{1+\sqrt{1-\rho^{2}}}{2}$

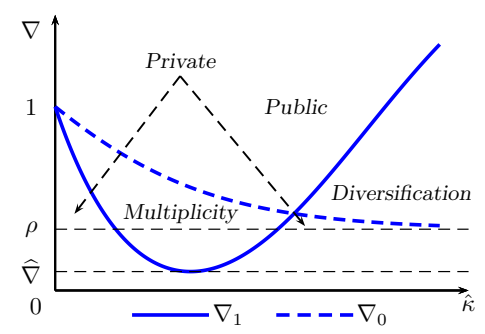

(e) $\frac{1+\sqrt{1-\rho^{2}}}{2}<\alpha<1$

Figure 6. Patterns of bounds $\nabla_{0}$ and $\nabla_{1}$.

Proof of Proposition 2. When $\hat{\kappa}$ is sufficiently large, $\nabla_{1}$ monotonically increases and $\lim _{\hat{\kappa} \rightarrow+\infty} \nabla_{1}=\frac{1}{\rho}$ while $\nabla_{0}$ monotonically decreases and $\lim _{\hat{\kappa} \rightarrow+\infty} \nabla_{0}=\rho$. Therefore, for any $\nabla$, when $\hat{\kappa}$ is sufficiently large, it holds that $\nabla \in\left(\nabla_{0}, \nabla_{1}\right)$. According to Proposition 1 , the equilibrium is unique and $0<\hat{\kappa}_{z}^{*}<\hat{\kappa}$. Further, the last part of this proposition can be obtained from equation (18).

Proof of Proposition 3. The complete characterization of the equilibrium attention allocation can be summarized in the following claims.

Claim 1: If the relative accuracy is sufficiently low (i.e., $\nabla<\hat{\nabla}$ ), agents specialize in learning the private signal and then eventually diversify their attention when the capacity increases, where

$$
\hat{\nabla}=\frac{1}{\rho}-\frac{1}{\rho} \frac{1}{2 \sqrt{\frac{\alpha(1-\alpha)}{\left(1-\rho^{2}\right)} \rho+\frac{2(1-\alpha)}{\left(1-\rho^{2}\right)} \rho^{2}+1}}
$$

$\hat{\kappa}_{z}^{*} / \hat{\kappa}$ can be either monotonically increasing or hump-shaped.

Proof. We first establish some properties of $\nabla_{0}$ and $\nabla_{1}$. As illustrated in Figure 6, there are in total five patterns, according to the combinations of $\alpha$ and $\rho$. For any $\hat{\kappa}>0$, the bounds $\nabla_{0}$ and $\nabla_{1}$ can be characterized as follows,

1. $\lim _{\hat{\kappa} \rightarrow+\infty} \nabla_{0}=\rho$ and $\lim _{\hat{\kappa} \rightarrow+\infty} \nabla_{1}=1 / \rho$. 
2. $\nabla_{0}(0)=\nabla_{1}(0)=1$.

3. $\nabla_{0}$ monotonically decreases in $\hat{\kappa}$.

4. $\nabla_{1}$ may or may not be monotone:

(i) If $0<\alpha<\frac{1-\rho}{2}, \nabla_{1}$ monotonically increases in $\hat{\kappa}$. Otherwise, $\nabla_{1}$ decreases and then increases, reaching the trough at $\hat{\kappa}=\hat{\kappa}$, where

$$
\hat{\kappa}=\ln \left(\rho+\sqrt{\alpha\left(1-\rho^{2}\right) /(1-\alpha)}\right) \text {. }
$$

(ii) If $\frac{1-\rho}{2}<\alpha<1-\rho, \nabla_{1}$ is always larger than $\nabla_{0}$ for any $\hat{\kappa}$.

(iii) If $1-\rho<\alpha, \nabla_{0}$ and $\nabla_{1}$ cross only once at $\hat{\kappa}=\ln \left(\frac{\rho}{1-\alpha}\right)$, on the condition that $\hat{\kappa}$ is positive. Further, $\nabla_{1}$ is smaller than $\nabla_{0}$ if and only if $\hat{\kappa}<\ln \left(\frac{\rho}{1-\alpha}\right)$.

(iv) If $1-\rho<\alpha<1-\rho^{2}, \nabla_{0}$ and $\nabla_{1}$ cross on the left side of $\hat{\hat{\kappa}}$; if $1-\rho^{2}<\alpha<1$, they cross on the right side of $\hat{\hat{\kappa}}$.

We can show the first three items by using the expression in equation (19). The last item can be verified by noting that,

$$
\frac{\mathrm{d} \nabla_{1}}{\mathrm{~d} \hat{\kappa}} \propto(1-\alpha) \exp (2 \hat{\kappa})-2(1-\alpha) \rho \exp (\hat{\kappa})+\rho^{2}-\alpha
$$

Denote $\hat{\nabla} \equiv \nabla_{1}(\hat{\hat{\kappa}})$ and $\tilde{\nabla} \equiv \nabla_{1}\left(\ln \left(\frac{\rho}{1-\alpha}\right)\right)$, we obtain

$$
\hat{\nabla}=\frac{1}{\rho}-\frac{1}{\rho} \frac{1}{2 \sqrt{\frac{\alpha(1-\alpha)}{\left(1-\rho^{2}\right)}} \rho+\frac{2(1-\alpha)}{\left(1-\rho^{2}\right)} \rho^{2}+1} ; \quad \tilde{\nabla}=\frac{1}{\rho}-\frac{1}{\rho} \frac{\alpha\left(1-\rho^{2}\right)}{1-(1-\alpha)^{2}}
$$

Intuitively, $\hat{\nabla}$ is the minimum of $\nabla_{1}$ and $\tilde{\nabla}$ is such that $\tilde{\nabla}=\nabla_{1}\left(\ln \left(\frac{\rho}{1-\alpha}\right)\right)=$ $\nabla_{0}\left(\ln \left(\frac{\rho}{1-\alpha}\right)\right)$. If $\nabla \in(\rho, \hat{\nabla})$, it holds that $\nabla<\nabla_{1}$ for any $\hat{\kappa}$; and there exists a cutoff $\hat{\kappa}_{0}$, such that for any $\hat{\kappa} \in\left(0, \hat{\kappa}_{0}\right), \nabla<\nabla_{0}$ and for any $\hat{\kappa} \geq \hat{\kappa}_{0}, \nabla \geq \nabla_{0}$. According to Proposition 1, the first part of this Claim is shown.

Regarding the pattern of attention allocation $\hat{\kappa}_{z}^{*} / \hat{\kappa}$, it can be categorized in the following two cases:

1. When the coordination motive is not so high, i.e., $0<\alpha<1-\rho, \hat{\kappa}_{z}^{*} / \hat{\kappa}$ is monotonically increasing in $\hat{\kappa}$, if $\nabla<\bar{\nabla}$, where $\bar{\nabla}=[\rho+(1-\alpha)] /[(1-\alpha) \rho+1]$; and is hump-shaped in $\hat{\kappa}$, if $\bar{\nabla}<\nabla<1$.

2. When the coordination motive is high, i.e., $1-\rho<\alpha, \hat{\kappa}_{z}^{*} / \hat{\kappa}$ is hump-shaped in $\hat{\kappa}$, if $\bar{\nabla}<\nabla<\min \{\tilde{\nabla}, \hat{\nabla}\}$; and is monotonically increasing in $\hat{\kappa}$, if $\nabla<\bar{\nabla}$. 
The details of the proof of the above two cases are contained in the Technical Appendix.

Claim 2: Suppose the coordination motive is strong, i.e., $(1-\rho) / 2<\alpha \leq 1-\rho$, and the relative accuracy is not extremely high, i.e., $\hat{\nabla}<\nabla<1$. Agents re-allocate their attention in the following fashion. When capacity is low, they specialize in learning the private signal only, then diversify their attention allocation and then specialize in learning the public signal only before eventually diversifying again.

Proof. The proof is similar to that of Claim 1. See Figure 6(b) for illustration.

Claim 3: Suppose the coordination motive is very strong, i.e., $1-\rho<\alpha<1$, and the relative accuracy is not extremely high, i.e., $\tilde{\nabla} \leq \nabla<1$. Agents re-allocate their attention in the following fashion. When the capacity is sufficiently low, they focus only on the private signal. When the capacity is higher, they may coordinate on one of the three equilibria. When there is a further increase in capacity, they pay attention only to the public signal. And when the capacity is sufficiently high, they eventually diversify.

Proof. We show that $\tilde{\nabla}<1$ if and only if $\rho>1-\alpha$. The proof is similar to that of Claim 1. See Figure 6(c), (d) and (e) for illustration.

Claim 4: Suppose the coordination motive is very strong, i.e., $1-\rho<\alpha \leq 1$, and the relative accuracy is low, i.e., $\max \{\rho, \hat{\nabla}\} \leq \nabla<\tilde{\nabla}$. If $1-\rho<\alpha \leq 1-\rho^{2}$, they allocate their attention in the same fashion as that in Claim 2. If $1-\rho^{2}<\alpha \leq 1$, when capacity is low, agents specialize in learning the private signal only. When the capacity is higher, they may coordinate on one of the three equilibria. When there is a further increase in capacity, they pay attention only to the private signal again. And when the capacity is sufficiently high, they eventually diversify. Note that $\rho<\hat{\nabla}$, if and only if $\alpha<\left(1+\sqrt{1-\rho^{2}}\right) / 2$.

Proof. The proof is similar to that of Claim 1. See Figure 6(d) and (e) for illustration.

Proof of Lemma 2. This proof offers a sufficient condition under which Lemma 2 holds. We consider the following two cases: (i) $2 \alpha+\rho>1,(1-\alpha) \exp (\hat{\kappa})>\rho$ and $\nabla<1$; (ii) $\alpha+\rho>1$ and $\nabla<\hat{\nabla}$. Under these two cases, if $\hat{\kappa}>\hat{\kappa}_{0}, 0 \leq \hat{\kappa}_{x}^{*}<\hat{\kappa}$. Therefore, we can 
show,

$$
\begin{aligned}
\frac{\mathrm{d} \hat{\kappa}_{x}^{*}}{\mathrm{~d} \hat{\kappa}} & \propto(\nabla-\rho)(1-\alpha) \exp (2 \hat{\kappa})-\left[\left(1-\rho^{2}\right)-(1-\rho \nabla)(1-\alpha)\right](1-\alpha) \exp (\hat{\kappa}) \\
& -((1-\alpha) \exp (\hat{\kappa})-\rho)\left[\left(1-\rho^{2}\right)-(1-\alpha)(1-\nabla \rho)\right] \\
& <\left[(\nabla-\rho) \exp (\hat{\kappa})-\left(1-\rho^{2}\right)+(1-\rho \nabla)(1-\alpha)\right](1-\alpha) \exp (\hat{\kappa}) .
\end{aligned}
$$

Therefore, $\mathrm{d} \hat{\kappa}_{x}^{*} / \mathrm{d} \hat{\kappa}<0$, if $\left[(\nabla-\rho) \exp (\hat{\kappa})-\left(1-\rho^{2}\right)+(1-\rho \nabla)(1-\alpha)\right]<0$. This holds true, if

$$
\frac{1-\nabla \rho}{\nabla-\rho}<\exp (\hat{\kappa})<\frac{\left(1-\rho^{2}\right)-(1-\rho \nabla)(1-\alpha)}{\nabla-\rho}
$$

The first inequality must hold so that $\hat{\kappa}>\hat{\kappa}_{0}$. The second inequality can hold on the condition that $\alpha$ is sufficiently large; that is,

$$
\alpha>1-\frac{\left(1-\rho^{2}\right)}{(1-\rho \nabla)} .
$$

Proof of Proposition 4. A simple calculation leads to

$$
\frac{\mathrm{d} \nabla_{1}}{\mathrm{~d} \rho}=\frac{(\exp (2 \hat{\kappa})-1)(1-\alpha)\left[-(1-\alpha)(\exp (2 \hat{\kappa})-1)+2 \rho \exp (\hat{\kappa})-\left(1+\rho^{2}\right)\right]}{\left[(1-\alpha)(\exp (2 \hat{\kappa})-1) \rho+\exp (\hat{\kappa})\left(1-\rho^{2}\right)\right]^{2}}
$$

Let $T_{1}=-(1-\alpha)(\exp (2 \hat{\kappa})-1)+2 \rho \exp (\hat{\kappa})-\left(1+\rho^{2}\right)$. If and only if $\rho<(1-$ $\alpha) \exp (\hat{\kappa}), T_{1}$ decreases in $\hat{\kappa}$ and $T_{1}<0$. In other words, $\mathrm{d} \nabla_{1} / \mathrm{d} \rho<0$ for any $\hat{\kappa}>$ $\ln (\rho /(1-\alpha))$. This implies that $\hat{\kappa}_{1}$ increases in $\rho$. Similarly, we can show that $\nabla_{0}$ is an increasing function of $\rho$, and therefore $\hat{\kappa}_{0}$ increases in $\rho$.

Let $l(\hat{\kappa})$ be the difference between the expected utility of adopting the strategy $\hat{\kappa}_{z}^{*}=\hat{\kappa}$ and that of $\hat{\kappa}_{z}^{*}=0$, when condition (20) holds. The cutoff $\hat{\kappa}_{s}$ is such that $l\left(\hat{\kappa}_{s}\right)=0$. It implies that $\hat{\kappa}_{z}^{*}=\hat{\kappa}$ if and only if

$$
l(\hat{\kappa})=\left[1+\frac{\left(1-\rho^{2}\right)}{\exp (2 \hat{\kappa})-1}\right] \nabla^{2}-\alpha \rho \nabla-\left[\frac{\left(1-\rho^{2}\right)}{\exp (2 \hat{\kappa})-1}+(1-\alpha)\right]>0 .
$$

Under this circumstance, it is straightforward to show the following facts: $l(\hat{\kappa})$ is strictly increasing in $\hat{\kappa}, \lim _{\hat{\kappa} \rightarrow 0} l(\hat{\kappa})<0$ and $\lim _{\hat{\kappa} \rightarrow+\infty} l(\hat{\kappa})>0$. Therefore, there is a 
unique $\hat{\kappa}_{s}>0$, such that $l\left(\hat{\kappa}_{s}\right)=0$, where

$$
\hat{\kappa}_{s}=\ln \left(\sqrt{\frac{\left(1-\rho^{2}\right)\left(1-\nabla^{2}\right)}{\alpha(1-\rho \nabla)-\left(1-\nabla^{2}\right)}+1}\right) .
$$

By noting that $\hat{\kappa}_{s}$ decreases in $\rho$, this proposition is shown.

Proof of Proposition 5. We show that social welfare can be decreasing in the case where $\alpha+\rho>1$ and $\tilde{\nabla}<\nabla<1$. If $\hat{\kappa}_{z}^{*}=0$ or $\hat{\kappa}_{z}^{*}=\hat{\kappa}$, social welfare is calculated by the following

$$
\begin{aligned}
& E\left[\mathcal{W}^{s}\right]=-\left[\frac{1+(1-\alpha) \frac{\phi_{x}}{\phi_{\theta}}}{\left(\frac{\phi_{x}}{\phi_{\theta}}+1\right)^{2}}\right] \sigma^{2}, \\
& E\left[\mathcal{W}^{s}\right]=-\left[\frac{1+(1-\alpha) \frac{\phi_{z}}{\phi_{\theta}}}{\left(\frac{\phi_{z}}{\phi_{\theta}}+1\right)^{2}}\right] \sigma^{2}-\alpha \frac{\left(\frac{\phi_{z}}{\phi_{\theta}}\right)^{2}}{\left(\frac{\phi_{z}}{\phi_{\theta}}+1\right)^{2}} \sigma_{z}^{2},
\end{aligned}
$$

when $\hat{\kappa}<\hat{\kappa}_{s}, \hat{\kappa}_{z}^{*}=0$ and $\mathrm{d} E[\mathcal{W}(\mathbf{a}, \theta)] / \mathrm{d} \hat{\kappa}>0$. To see this, we notice that $\phi_{x}$ increases in $\hat{\kappa}$ and $E\left[\mathcal{W}^{s}\right]$ increases in $\phi_{x}$,

$$
\frac{\mathrm{d} E\left[\mathcal{W}^{s}\right]}{\mathrm{d} \phi_{x}}=\frac{\sigma^{2}\left[(1+\alpha)+(1-\alpha) \frac{\phi_{x}}{\phi_{\theta}}\right]}{\left(1+\frac{\phi_{x}}{\phi_{\theta}}\right)^{3} \phi_{\theta}}>0 .
$$

Similarly, when $\hat{\kappa}_{s}<\hat{\kappa}<\hat{\kappa}_{1}, \hat{\kappa}_{z}^{*}=\hat{\kappa}$ and social welfare increases in $\hat{\kappa}$. When $\hat{\kappa}=\hat{\kappa}_{s}$, agents are indifferent of specialization in private or public signals, which implies that $\phi_{x}=\phi_{z}$, and social welfare discontinuously decreases at $\hat{\kappa}=\hat{\kappa}_{s}$. Because $E\left[\mathcal{W}^{s}\right]$ monotonically increases in $\hat{\kappa}$, when $\hat{\kappa}<\hat{\kappa}_{s}$, there must exist $\hat{\kappa}_{a}$ and $\hat{\kappa}_{b}$ such that $E\left[\mathcal{W}^{s}\left(\hat{\kappa}_{a}\right)\right]>$ $E\left[\mathcal{W}^{s}\left(\hat{\kappa}_{b}\right)\right]$ and $\hat{\kappa}_{a}<\hat{\kappa}_{s}<\hat{\kappa}_{b}$.

Proof of Proposition 6. Except in the case where $\alpha+\rho>1$ and $\tilde{\nabla}<\nabla<1$, we can show that $\hat{\kappa}_{x}^{*}=\hat{\kappa}_{0}$ and $\hat{\kappa}_{z}^{*}=0$, when $\hat{\kappa}=\hat{\kappa}_{0}$. To show $E\left[\mathcal{W}^{s}\left(\hat{\kappa}_{0}\right)\right]>E\left[\mathcal{W}^{m s}\right]$, we only need to show $f \equiv E\left[\mathcal{W}^{s}\left(\hat{\kappa}_{0}\right)\right]-E\left[\mathcal{W}^{m s}\right]>0$, where

$$
\begin{aligned}
f= & \frac{1}{\left(\frac{\rho}{(\nabla-\rho)}+\frac{1}{(1-\alpha)(1-\nabla \rho)}\right)}-\alpha \frac{\frac{\rho}{(\nabla-\rho)}}{\left(\frac{\rho}{(\nabla-\rho)}+\frac{1}{(1-\alpha)(1-\nabla \rho)}\right)^{2}} \\
& -\frac{1}{\left(\frac{\rho\left(1-\nabla^{2}\right)}{(1-\rho \nabla)(\nabla-\rho)}+\frac{1}{1-\alpha}\right)}-\alpha \frac{\frac{\rho\left(1-\nabla^{2}\right)}{(1-\rho \nabla)(\nabla-\rho)}}{\left(\frac{\rho\left(1-\nabla^{2}\right)}{(1-\rho \nabla)(\nabla-\rho)}+\frac{1}{1-\alpha}\right)^{2}} .
\end{aligned}
$$


To provide a sufficient condition under which the inequality holds, we denote

$$
f(\alpha)=f_{1}(\alpha)+f_{2}(\alpha),
$$

where

$$
\begin{aligned}
& f_{1}(\alpha)=\left(\frac{1}{1-\rho \nabla}-1\right)(1-\alpha)^{2}\left(-\alpha^{2}+4 \alpha-2\right)\left(\frac{\rho}{\nabla-\rho}\right)^{2} \\
& f_{2}(\alpha)=\frac{(1-\alpha)^{2} \rho \nabla}{1-\rho \nabla}\left[\left(\frac{1}{1-\rho \nabla}-1\right)(\alpha-2)^{2}+2\left(\frac{1}{1-\rho \nabla}-1\right)^{2}-2-\frac{1}{1-\rho \nabla} \frac{(2-\alpha)^{2}}{(1-\alpha)^{2}}\right]
\end{aligned}
$$

$f_{1}(\alpha)>0$ if and only if $-\alpha^{2}+4 \alpha-2>0$, or $\alpha>2-\sqrt{2}$. When $\nabla$ is sufficiently low and close to $\rho, \frac{\rho}{\nabla-\rho}$ can be arbitrarily large and $\frac{1}{1-\rho \nabla}$ is close to a constant $\frac{1}{1-\rho^{2}}$. Therefore, $f_{1}(\alpha)$ can be arbitrarily large and $f_{2}(\alpha)$ is close to a constant. Moreover, it must hold that $\rho<(1-\alpha) \exp \left(\hat{\kappa}_{0}\right)$ or,

$$
\frac{(1-\alpha)}{\rho}>\frac{\nabla-\rho}{1-\rho \nabla}
$$

This holds when $\nabla$ is low enough. 


\section{References}

Angeletos, G.-M. and A. Pavan (2006). Socially optimal coordination: Characterization and policy implication. Journal of the European Economic Association 5(2-3), pp. 585-593.

Angeletos, G.-M. and A. Pavan (2007). Efficient use of information and social value of information. Econometrica 75(4), pp. 1103-1142.

Colombo, L., G. Femminis, and A. Pavan (2012). Information acquisition and welfare. manuscript.

Cornand, C. and F. Heinemann (2008). Optimal degree of public information dissemination. The Economic Journal 118(528), pp. 718-742.

Hellwig, C., S. Kohls, and L. Veldkamp (2012). Information choice technologies. American Economic Review PEP 102(3), p.35-40.

Hellwig, C. and L. Veldkamp (2009). Knowing what others know: Coordination motives in information acquisition. Review of Economic Studies 76(1), 223-251.

Kahneman, D. (1973). Attention and Effort. Englewood Cliffs, New Jersey: PrenticeHall Inc.

Luo, Y. (2008). Consumption dynamics under information processing constraints. Review of Economic Dynamics 11(2), 366-385.

Luo, Y. and E. R. Young (2010). Risk-sensitive consumption and savings under rational inattention. American Economic Journal: Macroeconomics 2(4), 281-325.

Luo, Y. and E. R. Young (2014). Signal extraction and rational inattention, Economic Inquiry 52(2), 811-829.

Maćkowiak, B. A. and M. Wiederholt (2009). Optimal sticky prices under rational inattention. American Economic Review 99(3).

Maćkowiak, B. A. and M. Wiederholt (2011). Inattention to rare events. CEPR Discussion Papers 8626, C.E.P.R. Discussion Papers.

Ming, Y. (2013). Coordination with flexible information acquisition. manuscript, Princeton University Economic Theory Center.

Mondria, J. (2010). Portfolio choice, attention allocation, and price comovement. Journal of Economic Theory 145(5), 1837-1864.

Morris, S. and H. S. Shin (2002). Social value of public information. American Economic Review 92(5), 1521-1534.

Myatt, D. P. and C. Wallace (2009). On the sources and value of information: Public announcements and macroeconomic performance. Technical report. 
Myatt, D. P. and C. Wallace (2012). Endogenous information acquisition in coordination games. The Review of Economic Studies 79(1), 340-374.

Peng, L. (2005). Learning with information capacity constraints. Journal of Financial and Quantitative Analysis 40(02), 307-329.

Peng, L. and W. Xiong (2006). Investor attention, overconfidence and category learning. Journal of Financial Economics 80(3), 563-602.

Radner, R. (1962). Team decision problems. The Annals of Mathematical Statistics 33(3), 857-881.

Sims, C. A. (2003). Implications of rational inattention. Journal of Monetary Economics 50(3), 665-690.

Sims, C. A. (2005). Rational inattention: a research agenda. Technical report.

Sims, C. A. (2010). Rational inattention and monetary economics. In B. M. Friedman and M. Woodford (Eds.), Volume 3 of Handbook of Monetary Economics, Chapter 4, pp. 155-181. Elsevier.

Svensson, L. E. O. (2006). Social value of public information: Comment: Morris and shin (2002) is actually pro-transparency, not con. American Economic Review 96(1).

Van Nieuwerburgh, S. and L. Veldkamp (2009). Information immobility and the home bias puzzle. Journal of Finance 64(3), 1187-1215.

Van Nieuwerburgh, S. and L. Veldkamp (2010). Information acquisition and underdiversification. Review of Economic Studies 77(2), 779-805.

Veldkamp, L. (2011). Information choice in macroeconomics and finance / Laura L. Veldkamp. Princeton University Press, Princeton, N.J. 


\section{Technical Appendix}

(Not intended for publication)

Proof of Proposition 3, Part 2 of Claim 1. The following lemma is particularly useful for our characterization.

Lemma 3. When the equilibrium is unique, the fraction of attention paid to the public signal, i.e., $\hat{\kappa}_{z}^{*} / \hat{\kappa}$, strictly increases in $\hat{\kappa}$ if and only if $0<\hat{\kappa}_{z}^{*} / \hat{\kappa}<1$ and

$$
\frac{\hat{\kappa}_{z}^{*}}{\hat{\kappa}}-F(\hat{\kappa})<0,
$$

where

$$
\begin{aligned}
& F \equiv \frac{1}{2}+\frac{1}{2} \frac{T}{(1-\alpha)(1-\rho \nabla)(\nabla-\rho)} \cdot \frac{1}{1-\frac{\left(1-\rho^{2}\right)-(1-\alpha)(1-\rho \nabla)}{(\nabla-\rho) \exp (\hat{\kappa})}} \cdot \frac{1}{\exp (\hat{\kappa})-\frac{\rho}{1-\alpha}}, \\
& T=(1-\rho \nabla) \alpha[(1-\rho \nabla)(1-\alpha)-\rho(\nabla-\rho)] .
\end{aligned}
$$

Proof. We re-write equation (18) as follows,

$$
\frac{\hat{\kappa}_{z}^{*}}{\hat{\kappa}}=\frac{1}{2}+\frac{\frac{1}{2} \ln \left[\frac{(1-\alpha)(1-\rho \nabla)+(\nabla-\rho) \exp (\hat{\kappa})-\left(1-\rho^{2}\right)}{(1-\alpha)(1-\rho \nabla) \exp (\hat{\kappa})+(\nabla-\rho)-\nabla\left(1-\rho^{2}\right)}\right]}{\ln [\exp (\hat{\kappa})]} .
$$

Derive its derivative with respect to $\exp (\hat{\kappa})$ and we find that $\partial\left(\hat{\kappa}_{z}^{*} / \hat{\kappa}\right) / \partial \exp (\hat{\kappa})>0$ if and only if the condition (26) holds.

We first show that in the case of $\nabla>1, \hat{\kappa}_{z}^{*} / \hat{\kappa}$ monotonically decreases in $\hat{\kappa}$, when $\hat{\kappa}>\hat{\kappa}_{1}$. There are three sub-cases.

Case 1: $\alpha<1-\rho$ and $\nabla \in\left(\frac{1-\alpha+\rho^{2}}{(1-\alpha) \rho+\rho}, \frac{1}{\rho}\right)$. We can verify that $T<0$ and further

$$
0>\ln \left(\frac{\rho}{1-\alpha}\right)>\ln \left(\frac{\left(1-\rho^{2}\right)-(1-\alpha)(1-\rho \nabla)}{\nabla-\rho}\right)
$$

which implies that $F(\hat{\kappa})$ is strictly increasing and approaches $1 / 2$ from below when $\hat{\kappa}$ approaches $+\infty$.

We can also show that $\hat{\kappa}_{z}^{*} / \hat{\kappa}$ approaches $1 / 2$ from above, because the second term 
in (27) is positive when $\hat{\kappa}$ approaches $+\infty$, i.e.,

$$
\begin{aligned}
& \lim _{\hat{\kappa} \rightarrow+\infty} \ln \left[\frac{(1-\alpha)(1-\rho \nabla)+(\nabla-\rho) \exp (\hat{\kappa})-\left(1-\rho^{2}\right)}{(1-\alpha)(1-\rho \nabla) \exp (\hat{\kappa})+(\nabla-\rho)-\nabla\left(1-\rho^{2}\right)}\right] \\
= & \ln \left[\frac{\nabla-\rho}{(1-\alpha)(1-\rho \nabla)}\right]>0 .
\end{aligned}
$$

Further, the first part of Proposition 3 implies that $\lim _{\hat{\kappa} \rightarrow \hat{\kappa}_{1}^{+}} \hat{\kappa}_{z}^{*} / \hat{\kappa}=1$. We can show that $\hat{\kappa}_{z}^{*} / \hat{\kappa}-F(\hat{\kappa})>0$, for any $\hat{\kappa}>\hat{\kappa}_{1}$, by constructing a contradiction. Suppose there exists $\hat{\kappa}^{\prime}$ such that $\hat{\kappa}_{z}^{*}\left(\hat{\kappa}^{\prime}\right) / \hat{\kappa}^{\prime}<F\left(\hat{\kappa}^{\prime}\right)$. Lemma 3 implies that it must hold that $\hat{\kappa}_{z}^{*} / \hat{\kappa}$ approaches $1 / 2$ from below. A contradiction. This fact further implies that $\hat{\kappa}_{z}^{*} / \hat{\kappa}$ monotonically decreases, by using Lemma 3 again.

Case 2: $\alpha<1-\rho$ and $\nabla \in\left(1, \frac{1-\alpha+\rho^{2}}{(1-\alpha) \rho+\rho}\right)$. In this case, we can show that $F(\hat{\kappa})$ is strictly decreasing and approaches $1 / 2$ from above, when $\hat{\kappa}$ approaches $+\infty$. Further, it must hold that $F\left(\hat{\kappa}_{1}\right) \leq 1$, that is because $\hat{\kappa}_{z}^{*} / \hat{\kappa}$ must decrease, when $\hat{\kappa}$ is slightly higher than $\hat{\kappa}_{1}$, according to the first part of Proposition 3.

Similar to the previous case, Lemma 3 implies that $\hat{\kappa}_{z}^{*} / \hat{\kappa}-F(\hat{\kappa})$ cannot cross zero from above; that is, $\hat{\kappa}_{z}^{*} / \hat{\kappa}-F(\hat{\kappa})>0$ for any $\hat{\kappa}>\hat{\kappa}_{1}$. In other words, $\hat{\kappa}_{z}^{*} / \hat{\kappa}$ decreases monotonically.

Case 3: The proof for the case where $\alpha>1-\rho$ and $\nabla>1$ is similar.

We then establish that $\hat{\kappa}_{z}^{*} / \hat{\kappa}$ can be either monotonically increasing or hump-shaped when $\nabla<1$.

Case 1: $\alpha<1-\rho$ and $\nabla \in(\rho, \bar{\nabla})$. In this case, we can show that $F(\hat{\kappa})$ is monotonically decreasing and approaches $1 / 2$ from above. The first part of Proposition 3 implies that $\lim _{\hat{\kappa} \rightarrow \hat{\kappa}_{0}^{+}} \hat{\kappa}_{z}^{*} / \hat{\kappa}=0$. Under this case, $\hat{\kappa}_{z}^{*} / \hat{\kappa}$ approaches $1 / 2$ from below because the second term in (27) is negative when $\hat{\kappa}$ approaches $+\infty$, i.e.,

$$
\begin{aligned}
& \lim _{\hat{\kappa} \rightarrow+\infty} \ln \left[\frac{(1-\alpha)(1-\rho \nabla)+(\nabla-\rho) \exp (\hat{\kappa})-\left(1-\rho^{2}\right)}{(1-\alpha)(1-\rho \nabla) \exp (\hat{\kappa})+(\nabla-\rho)-\nabla\left(1-\rho^{2}\right)}\right] \\
= & \ln \left[\frac{\nabla-\rho}{(1-\alpha)(1-\rho \nabla)}\right]<0 .
\end{aligned}
$$

Using similar arguments in previous cases, we can show that $\hat{\kappa}_{z}^{*} / \hat{\kappa}$ monotonically increases in $\hat{\kappa}$.

Case 2: $\alpha \in(0,1-\rho)$ and $\nabla \in(\bar{\nabla}, 1)$. This case differs from the previous one in that $\hat{\kappa}_{z}^{*} / \hat{\kappa}$ approaches $1 / 2$ from above when $\hat{\kappa} \rightarrow+\infty$. Therefore, Lemma 3 implies that $\hat{\kappa}_{z}^{*} / \hat{\kappa}$ must be increasing and then decreasing, i.e., it is hump-shaped.

The proofs of the remaining cases are also similar. 